\title{
Lipid profiling and microstructure characteristics of goat milk fat from different stages of
}

lactation

Yan Zhang†, Zhaojun Zheng†, Chunhuan Liu, Yuanfa Liu*

State Key Laboratory of Food Science and Technology, School of Food Science and Technology, National Engineering Research Center for Functional Food, National Engineering Laboratory for Cereal Fermentation Technology, Collaborative Innovation Center of Food Safety and Quality

Control in Jiangsu Province, Jiangnan University, 1800 Lihu Road, Wuxi, Jiangsu 214122, People's Republic of China

*Corresponding author: Yuanfa Liu

Phone: 0510-85876799; Fax: 0510-85876799; E-mail: yfliu@jiangnan.edu.cn

$\dagger$ Authors contributed equally

Declarations of interest: none 
Table S1. Composition and relative content (\%) of triacylglycerols (TAGs) identified in goat colostrum, transitional and mature milk. ${ }^{a}$

\begin{tabular}{|c|c|c|c|c|c|c|c|c|c|}
\hline Number & $\begin{array}{l}\text { Retention } \\
\text { time (min) }\end{array}$ & {$\left[\mathrm{M}+\mathrm{NH}_{4}\right]^{+; b}$} & Formula & $\begin{array}{l}\text { ACN: } \\
\text { DB }\end{array}$ & TAGs $^{c}$ & $\begin{array}{l}\text { Mass } \\
\text { Error } \\
(\mathrm{ppm})\end{array}$ & Colostrum & Transitional & Mature \\
\hline 1 & 8.03 & 650.5355 & $\mathrm{C}_{39} \mathrm{H}_{68} \mathrm{O}_{6}$ & $36: 3$ & $\mathrm{MLnBu}$ & 0.2 & $0.067 \pm 0.001$ & $0.057 \pm 0.001$ & $0.054 \pm 0.001$ \\
\hline 2 & 8.22 & 676.5509 & $\mathrm{C}_{41} \mathrm{H}_{70} \mathrm{O}_{6}$ & $38: 4$ & BuPoLn & -0.3 & $0.008 \pm 0.000$ & $0.006 \pm 0.000$ & $0.005 \pm 0.000$ \\
\hline 3 & 8.37 & 702.5668 & $\mathrm{C}_{43} \mathrm{H}_{72} \mathrm{O}_{6}$ & $40: 5$ & BuLLn & 0.1 & $0.008 \pm 0.000$ & $0.007 \pm 0.001$ & $0.006 \pm 0.000$ \\
\hline 4 & 8.52 & 676.5514 & $\mathrm{C}_{41} \mathrm{H}_{70} \mathrm{O}_{6}$ & $38: 4$ & BuMARA & 0.5 & $0.018 \pm 0.001$ & $0.018 \pm 0.001$ & $0.012 \pm 0.001$ \\
\hline 5 & 8.53 & 664.5514 & $\mathrm{C}_{40} \mathrm{H}_{70} \mathrm{O}_{6}$ & $37: 3$ & BuPaLn & 0.5 & $0.005 \pm 0.002$ & $0.005 \pm 0.000$ & $0.004 \pm 0.002$ \\
\hline 6 & 8.58 & 702.5672 & $\mathrm{C}_{43} \mathrm{H}_{72} \mathrm{O}_{6}$ & $40: 5$ & BuMDp & 0.7 & $0.015 \pm 0.000$ & $0.007 \pm 0.000$ & $0.005 \pm 0.000$ \\
\hline 7 & 8.84 & 702.5672 & $\mathrm{C}_{43} \mathrm{H}_{72} \mathrm{O}_{6}$ & $40: 5$ & BuPEPA & 0.7 & $0.011 \pm 0.000$ & $0.007 \pm 0.000$ & $0.005 \pm 0.000$ \\
\hline 8 & 8.85 & 728.5826 & $\mathrm{C}_{45} \mathrm{H}_{74} \mathrm{O}_{6}$ & $42: 6$ & BuLARA & 0.4 & $0.005 \pm 0.001$ & $0.004 \pm 0.001$ & $0.002 \pm 0.000$ \\
\hline 9 & 9.01 & 678.5676 & $\mathrm{C}_{41} \mathrm{H}_{72} \mathrm{O}_{6}$ & $38: 3$ & BuPLn & 1.3 & $0.180 \pm 0.003$ & $0.167 \pm 0.006$ & $0.143 \pm 0.001$ \\
\hline 10 & 9.16 & 704.5832 & $\mathrm{C}_{43} \mathrm{H}_{74} \mathrm{O}_{6}$ & $40: 4$ & $\mathrm{BuOLn}+\mathrm{BuLL}$ & 1.2 & $0.070 \pm 0.009$ & $0.080 \pm 0.003$ & $0.054 \pm 0.002$ \\
\hline 11 & 9.25 & 728.5825 & $\mathrm{C}_{45} \mathrm{H}_{74} \mathrm{O}_{6}$ & $42: 6$ & BuPDHA & 0.2 & $0.008 \pm 0.000$ & $0.006 \pm 0.000$ & $0.004 \pm 0.000$ \\
\hline 12 & 9.30 & 666.5672 & $\mathrm{C}_{40} \mathrm{H}_{72} \mathrm{O}_{6}$ & $37: 2$ & $\mathrm{BuPaL}+\mathrm{BuPoMo}$ & 0.7 & $0.055 \pm 0.001$ & $0.053 \pm 0.002$ & $0.053 \pm 0.001$ \\
\hline 13 & 9.45 & 692.5829 & $\mathrm{C}_{42} \mathrm{H}_{74} \mathrm{O}_{6}$ & $39: 3$ & $\begin{array}{l}\text { BuMoL+BuMaLn+ } \\
\text { BuHdO }\end{array}$ & 0.7 & $0.026 \pm 0.000$ & $0.027 \pm 0.001$ & $0.018 \pm 0.000$ \\
\hline 14 & 9.59 & 704.5828 & $\mathrm{C}_{43} \mathrm{H}_{74} \mathrm{O}_{6}$ & $40: 4$ & BuPARA & 0.6 & $0.070 \pm 0.000$ & $0.050 \pm 0.010$ & $0.035 \pm 0.003$ \\
\hline 15 & 9.60 & 654.5677 & $\mathrm{C}_{39} \mathrm{H}_{72} \mathrm{O}_{6}$ & $36: 1$ & $\mathrm{MOBu}$ & 1.5 & $3.476 \pm 0.026$ & $4.589 \pm 0.025$ & $4.328 \pm 0.008$ \\
\hline 16 & 9.64 & 730.5993 & $\mathrm{C}_{45} \mathrm{H}_{76} \mathrm{O}_{6}$ & $42: 5$ & BuPDp & 1.7 & $0.126 \pm 0.001$ & $0.070 \pm 0.004$ & $0.046 \pm 0.001$ \\
\hline 17 & 9.77 & 756.6142 & $\mathrm{C}_{47} \mathrm{H}_{78} \mathrm{O}_{6}$ & $44: 6$ & $\mathrm{BuODp}$ & 0.6 & $0.014 \pm 0.000$ & $0.012 \pm 0.001$ & $0.007 \pm 0.000$ \\
\hline 18 & 9.83 & 680.583 & $\mathrm{C}_{41} \mathrm{H}_{74} \mathrm{O}_{6}$ & $38: 2$ & $\mathrm{BuPL}$ & 1.0 & $1.164 \pm 0.007$ & $1.409 \pm 0.060$ & $1.204 \pm 0.029$ \\
\hline 19 & 9.97 & 706.5991 & $\mathrm{C}_{43} \mathrm{H}_{76} \mathrm{O}_{6}$ & $40: 3$ & $\mathrm{BuOL}$ & 1.6 & $0.468 \pm 0.005$ & $0.616 \pm 0.025$ & $0.445 \pm 0.010$ \\
\hline 20 & 9.99 & 718.5985 & $\mathrm{C}_{44} \mathrm{H}_{76} \mathrm{O}_{6}$ & $41: 4$ & BuMaARA & 0.7 & $0.005 \pm 0.000$ & $0.004 \pm 0.000$ & $0.002 \pm 0.000$ \\
\hline 21 & 10.05 & 744.6143 & $\mathrm{C}_{46} \mathrm{H}_{78} \mathrm{O}_{6}$ & $43: 5$ & $\mathrm{DpMaBu}$ & 0.9 & $0.005 \pm 0.001$ & $0.002 \pm 0.001$ & $0.003 \pm 0.001$ \\
\hline 22 & 10.16 & 668.5834 & $\mathrm{C}_{40} \mathrm{H}_{74} \mathrm{O}_{6}$ & $37: 1$ & $\mathrm{BuPMo}+\mathrm{BuPaO}$ & 1.5 & $0.371 \pm 0.005$ & $0.436 \pm 0.018$ & $0.378 \pm 0.006$ \\
\hline 23 & 10.17 & 732.6143 & $\mathrm{C}_{45} \mathrm{H}_{78} \mathrm{O}_{6}$ & $42: 4$ & $\mathrm{LLCo}+\mathrm{CoOLn}$ & 0.9 & $0.041 \pm 0.001$ & $0.046 \pm 0.002$ & $0.035 \pm 0.001$ \\
\hline 24 & 10.24 & 756.6144 & $\mathrm{C}_{47} \mathrm{H}_{78} \mathrm{O}_{6}$ & $44: 6$ & CoPDHA & 0.9 & $0.004 \pm 0.000$ & $0.004 \pm 0.000$ & $0.003 \pm 0.000$ \\
\hline 25 & 10.32 & 694.5986 & $\mathrm{C}_{42} \mathrm{H}_{76} \mathrm{O}_{6}$ & $39: 2$ & $\begin{array}{l}\mathrm{BuMoO}+\mathrm{CoPaL}+\mathrm{Bu} \\
\mathrm{MaL}\end{array}$ & 0.8 & $0.109 \pm 0.001$ & $0.158 \pm 0.007$ & $0.101 \pm 0.002$ \\
\hline 26 & 10.50 & 720.6142 & $\mathrm{C}_{44} \mathrm{H}_{78} \mathrm{O}_{6}$ & $41: 3$ & $\begin{array}{l}\mathrm{VOL}+\mathrm{CoMaLn}+\mathrm{Co} \\
\mathrm{MoL}+\mathrm{CoHdO}\end{array}$ & 0.7 & $0.025 \pm 0.002$ & $0.024 \pm 0.001$ & $0.019 \pm 0.001$ \\
\hline 27 & 10.66 & 758.6298 & $\mathrm{C}_{47} \mathrm{H}_{80} \mathrm{O}_{6}$ & $44: 5$ & CoPDp & 0.6 & $0.098 \pm 0.001$ & $0.064 \pm 0.002$ & $0.046 \pm 0.002$ \\
\hline 28 & 10.72 & 682.5988 & $\mathrm{C}_{41} \mathrm{H}_{76} \mathrm{O}_{6}$ & $38: 1$ & $\mathrm{BuPO}$ & 1.1 & $5.626 \pm 0.048$ & $5.413 \pm 1.751$ & $7.082 \pm 0.104$ \\
\hline 29 & 10.78 & 784.6445 & $\mathrm{C}_{49} \mathrm{H}_{82} \mathrm{O}_{6}$ & $46: 6$ & ODpCo & -0.6 & $0.007 \pm 0.001$ & $0.007 \pm 0.001$ & $0.005 \pm 0.000$ \\
\hline 30 & 10.87 & 708.6149 & $\mathrm{C}_{43} \mathrm{H}_{78} \mathrm{O}_{6}$ & $40: 2$ & $\mathrm{BuOO}+\mathrm{CoPL}$ & 1.8 & $2.095 \pm 0.038$ & $3.298 \pm 0.096$ & $2.264 \pm 0.044$ \\
\hline 31 & 11.04 & 670.5986 & $\mathrm{C}_{40} \mathrm{H}_{76} \mathrm{O}_{6}$ & $37: 0$ & $\begin{array}{l}\mathrm{MaMCo}+\mathrm{MaPBu}+ \\
\mathrm{MaCaCa}\end{array}$ & 0.9 & $0.818 \pm 0.033$ & $0.798 \pm 0.106$ & $0.904 \pm 0.054$ \\
\hline 32 & 11.06 & 734.63 & $\mathrm{C}_{45} \mathrm{H}_{80} \mathrm{O}_{6}$ & $42: 3$ & $\mathrm{OLCo}+\mathrm{CaMLn}$ & 1.0 & $0.315 \pm 0.006$ & $0.377 \pm 0.018$ & $0.293 \pm 0.003$ \\
\hline
\end{tabular}




\begin{tabular}{|c|c|c|c|c|c|c|c|c|c|}
\hline 33 & 11.20 & 696.6143 & $\mathrm{C}_{42} \mathrm{H}_{78} \mathrm{O}_{6}$ & $39: 1$ & $\begin{array}{l}\mathrm{BuMaO}+\mathrm{CoPMo}+\mathrm{C} \\
\text { oPaO+CaLaMo }\end{array}$ & 0.9 & $0.457 \pm 0.004$ & $0.609 \pm 0.019$ & $0.464 \pm 0.004$ \\
\hline 34 & 11.24 & 760.6459 & $\mathrm{C}_{47} \mathrm{H}_{82} \mathrm{O}_{6}$ & $44: 4$ & $\begin{array}{l}\mathrm{CaPoLn}+\mathrm{CyOLn}+\mathrm{C} \\
\mathrm{yLL}\end{array}$ & 1.2 & $0.030 \pm 0.003$ & $0.032 \pm 0.002$ & $0.026 \pm 0.001$ \\
\hline 35 & 11.39 & 722.6299 & $\mathrm{C}_{44} \mathrm{H}_{80} \mathrm{O}_{6}$ & $41: 2$ & $\begin{array}{l}\mathrm{VOO}+\mathrm{VMoNe}+\mathrm{Co} \\
\mathrm{MoO}+\mathrm{CoMaL}+\mathrm{CyP} \\
\mathrm{aL}\end{array}$ & 0.7 & $0.083 \pm 0.001$ & $0.107 \pm 0.004$ & $0.078 \pm 0.001$ \\
\hline 36 & 11.61 & 748.6454 & $\mathrm{C}_{46} \mathrm{H}_{82} \mathrm{O}_{6}$ & $43: 3$ & $\begin{array}{l}\text { CyMoL+HpOL+Ca } \\
\text { PaLn }\end{array}$ & 0.6 & $0.023 \pm 0.000$ & $0.019 \pm 0.001$ & $0.018 \pm 0.000$ \\
\hline 37 & 11.63 & 684.6147 & $\mathrm{C}_{41} \mathrm{H}_{78} \mathrm{O}_{6}$ & $38: 0$ & $\mathrm{PMCy}+\mathrm{MCaM}$ & 1.4 & $5.629 \pm 0.103$ & $6.148 \pm 0.081$ & $7.366 \pm 0.085$ \\
\hline 38 & 11.69 & 760.6458 & $\mathrm{C}_{47} \mathrm{H}_{82} \mathrm{O}_{6}$ & $44: 4$ & CyPARA & 1.1 & $0.058 \pm 0.000$ & $0.044 \pm 0.002$ & $0.035 \pm 0.000$ \\
\hline 39 & 11.73 & 786.6612 & $\mathrm{C}_{49} \mathrm{H}_{84} \mathrm{O}_{6}$ & $46: 5$ & $\mathrm{CyPDp}+\mathrm{CaMDp}$ & 0.7 & $0.052 \pm 0.000$ & $0.032 \pm 0.002$ & $0.023 \pm 0.000$ \\
\hline 40 & 11.78 & 710.6304 & $\mathrm{C}_{43} \mathrm{H}_{80} \mathrm{O}_{6}$ & $40: 1$ & $\begin{array}{l}\mathrm{CoPO}+\mathrm{CyMO}+\mathrm{CaL} \\
\mathrm{aO}\end{array}$ & 1.6 & $4.859 \pm 0.057$ & $5.900 \pm 0.045$ & $6.050 \pm 0.040$ \\
\hline 41 & 11.92 & 696.6143 & $\mathrm{C}_{42} \mathrm{H}_{78} \mathrm{O}_{6}$ & $39: 1$ & СуMМо & 0.9 & $0.010 \pm 0.001$ & $0.008 \pm 0.001$ & $0.015 \pm 0.000$ \\
\hline 42 & 11.94 & 736.6463 & $\mathrm{C}_{45} \mathrm{H}_{82} \mathrm{O}_{6}$ & $42: 2$ & $\begin{array}{l}\mathrm{OOCo}+\mathrm{CaML}+\mathrm{CyP} \\
\mathrm{L}\end{array}$ & 1.8 & $1.485 \pm 0.013$ & $2.138 \pm 0.075$ & $1.603 \pm 0.019$ \\
\hline 43 & 11.97 & 812.6765 & $\mathrm{C}_{51} \mathrm{H}_{86} \mathrm{O}_{6}$ & $48: 6$ & $\mathrm{CaPoDp}+\mathrm{CyODp}$ & 0.3 & $0.005 \pm 0.000$ & $0.004 \pm 0.000$ & $0.003 \pm 0.000$ \\
\hline 44 & 11.99 & 786.6604 & $\mathrm{C}_{49} \mathrm{H}_{84} \mathrm{O}_{6}$ & $46: 5$ & TdTdEPA & -0.3 & $0.015 \pm 0.000$ & $0.010 \pm 0.000$ & $0.008 \pm 0.000$ \\
\hline 45 & 12.18 & 698.6298 & $\mathrm{C}_{42} \mathrm{H}_{80} \mathrm{O}_{6}$ & $39: 0$ & $\begin{array}{l}\mathrm{BuMaS}+\mathrm{CaMPa}+\mathrm{Ca} \\
\mathrm{LaMa}+\mathrm{CoPMa}+\mathrm{CoP} \\
\mathrm{aS}+\mathrm{CyPaP}+\mathrm{CyMMa}\end{array}$ & 0.7 & $0.661 \pm 0.002$ & $0.675 \pm 0.025$ & $0.740 \pm 0.008$ \\
\hline 46 & 12.19 & 762.6613 & $\mathrm{C}_{47} \mathrm{H}_{84} \mathrm{O}_{6}$ & $44: 3$ & $\begin{array}{l}\text { CaPoL+LaMLn+Cy } \\
\mathrm{OL}+\mathrm{CaPLn}+\mathrm{DcPL}\end{array}$ & 0.9 & $0.285 \pm 0.008$ & $0.291 \pm 0.008$ & $0.262 \pm 0.004$ \\
\hline 47 & 12.29 & 800.6767 & $\mathrm{C}_{50} \mathrm{H}_{86} \mathrm{O}_{6}$ & $47: 5$ & PePDp & 0.5 & $0.004 \pm 0.000$ & $0.002 \pm 0.000$ & $0.002 \pm 0.000$ \\
\hline 48 & 12.32 & 724.6458 & $\mathrm{C}_{44} \mathrm{H}_{82} \mathrm{O}_{6}$ & $41: 1$ & $\begin{array}{l}\mathrm{CoMaO}+\mathrm{CyPMo}+\mathrm{C} \\
\mathrm{yPaO}+\mathrm{CaMMo}\end{array}$ & 1.1 & $0.360 \pm 0.004$ & $0.421 \pm 0.014$ & $0.367 \pm 0.005$ \\
\hline 49 & 12.34 & 788.6778 & $\mathrm{C}_{49} \mathrm{H}_{86} \mathrm{O}_{6}$ & $46: 4$ & $\mathrm{CaOLn}+\mathrm{CaLL}$ & 1.9 & $0.078 \pm 0.001$ & $0.067 \pm 0.002$ & $0.061 \pm 0.001$ \\
\hline 50 & 12.43 & 812.6768 & $\mathrm{C}_{51} \mathrm{H}_{86} \mathrm{O}_{6}$ & $48: 6$ & CaPDHA & 0.6 & $0.005 \pm 0.000$ & $0.004 \pm 0.000$ & $0.003 \pm 0.000$ \\
\hline 51 & 12.50 & 750.6613 & $\mathrm{C}_{46} \mathrm{H}_{84} \mathrm{O}_{6}$ & $43: 2$ & $\begin{array}{l}\text { CyMoO+PeMoMo+ } \\
\text { HpOO+PePL+CaPa } \\
\text { L }\end{array}$ & 0.9 & $0.087 \pm 0.000$ & $0.092 \pm 0.004$ & $0.083 \pm 0.001$ \\
\hline 52 & 12.71 & 776.6759 & $\mathrm{C}_{48} \mathrm{H}_{86} \mathrm{O}_{6}$ & $45: 3$ & $\begin{array}{l}\mathrm{LaPaLn}+\mathrm{CaMaLn}+ \\
\mathrm{CaMoL}+\mathrm{PeOL}\end{array}$ & -0.5 & $0.026 \pm 0.001$ & $0.022 \pm 0.001$ & $0.020 \pm 0.001$ \\
\hline 53 & 12.75 & 712.6458 & $\mathrm{C}_{43} \mathrm{H}_{82} \mathrm{O}_{6}$ & $40: 0$ & $\begin{array}{l}\text { CoPS+CyMS+CyPP } \\
+\mathrm{CaMP}+\mathrm{CaLaS}+\mathrm{La} \\
\mathrm{MM}\end{array}$ & 1.2 & $4.644 \pm 0.057$ & $4.784 \pm 0.021$ & $5.839 \pm 0.052$ \\
\hline 54 & 12.82 & 788.677 & $\mathrm{C}_{49} \mathrm{H}_{86} \mathrm{O}_{6}$ & $46: 4$ & CaPARA & 0.9 & $0.065 \pm 0.001$ & $0.040 \pm 0.002$ & $0.036 \pm 0.000$ \\
\hline 55 & 12.84 & 814.6936 & $\mathrm{C}_{51} \mathrm{H}_{88} \mathrm{O}_{6}$ & $48: 5$ & CaPDp & 2.0 & $0.088 \pm 0.003$ & $0.045 \pm 0.001$ & $0.037 \pm 0.002$ \\
\hline 56 & 12.88 & 738.662 & $\mathrm{C}_{45} \mathrm{H}_{84} \mathrm{O}_{6}$ & $42: 1$ & $\begin{array}{l}\mathrm{CaMO}+\mathrm{LaLaO}+\mathrm{CyP} \\
\mathrm{O}\end{array}$ & 1.9 & $4.210 \pm 0.038$ & $4.612 \pm 0.046$ & $5.027 \pm 0.036$ \\
\hline 57 & 12.95 & 840.7089 & $\mathrm{C}_{53} \mathrm{H}_{90} \mathrm{O}_{6}$ & $50: 6$ & $\mathrm{CaODp}$ & 1.6 & $0.009 \pm 0.000$ & $0.006 \pm 0.000$ & $0.004 \pm 0.000$ \\
\hline 58 & 13.05 & 724.6453 & $\mathrm{C}_{44} \mathrm{H}_{82} \mathrm{O}_{6}$ & $41: 1$ & LaLaMo & 0.5 & $0.009 \pm 0.000$ & $0.007 \pm 0.000$ & $0.013 \pm 0.000$ \\
\hline 59 & 13.07 & 764.6779 & $\mathrm{C}_{47} \mathrm{H}_{86} \mathrm{O}_{6}$ & $44: 2$ & $\begin{array}{l}\mathrm{CaPL}+\mathrm{LaML}+\mathrm{LaPo} \\
\mathrm{Po}+\mathrm{CyOO}+\mathrm{CaPoO}\end{array}$ & 2.1 & $1.631 \pm 0.018$ & $1.921 \pm 0.072$ & $1.643 \pm 0.038$ \\
\hline 60 & 13.15 & 726.6618 & $\mathrm{C}_{44} \mathrm{H}_{84} \mathrm{O}_{6}$ & $41: 0$ & $\begin{array}{l}\mathrm{CaMMa}+\mathrm{CaPaP}+\mathrm{La} \\
\mathrm{MPa}+\mathrm{LaLaMa}\end{array}$ & 1.6 & $0.685 \pm 0.010$ & $0.580 \pm 0.017$ & $0.713 \pm 0.013$ \\
\hline 61 & 13.24 & 790.6936 & $\mathrm{C}_{49} \mathrm{H}_{88} \mathrm{O}_{6}$ & $46: 3$ & $\mathrm{CaOL}$ & 2.1 & $0.469 \pm 0.006$ & $0.464 \pm 0.020$ & $0.432 \pm 0.006$ \\
\hline 62 & 13.43 & 752.6779 & $\mathrm{C}_{46} \mathrm{H}_{86} \mathrm{O}_{6}$ & $43: 1$ & $\begin{array}{l}\mathrm{PePO}+\mathrm{CaPaO}+\mathrm{CaP} \\
\mathrm{Mo}+\mathrm{LaMMo}\end{array}$ & 2.2 & $0.424 \pm 0.005$ & $0.422 \pm 0.013$ & $0.420 \pm 0.002$ \\
\hline 63 & 13.45 & 816.7087 & $\mathrm{C}_{51} \mathrm{H}_{90} \mathrm{O}_{6}$ & $48: 4$ & $\mathrm{LaOLn}+\mathrm{LaLL}$ & 1.3 & $0.065 \pm 0.001$ & $0.039 \pm 0.002$ & $0.039 \pm 0.001$ \\
\hline 64 & 13.57 & 778.6925 & $\mathrm{C}_{48} \mathrm{H}_{88} \mathrm{O}_{6}$ & $45: 2$ & $\mathrm{LaPaL}+\mathrm{CaMoO}$ & 0.7 & $0.114 \pm 0.002$ & $0.118 \pm 0.006$ & $0.102 \pm 0.001$ \\
\hline
\end{tabular}




\begin{tabular}{|c|c|c|c|c|c|c|c|c|c|}
\hline 65 & 13.81 & 804.708 & $\mathrm{C}_{50} \mathrm{H}_{90} \mathrm{O}_{6}$ & $47: 3$ & $\begin{array}{l}\mathrm{LaMoL}+\mathrm{MPaLn}+\mathrm{La} \\
\text { MaLn }\end{array}$ & 0.5 & $0.024 \pm 0.001$ & $0.016 \pm 0.001$ & $0.016 \pm 0.000$ \\
\hline 66 & 13.87 & 740.6774 & $\mathrm{C}_{45} \mathrm{H}_{86} \mathrm{O}_{6}$ & $42: 0$ & $\begin{array}{l}\text { CaMS+LaMP+LaLa } \\
\text { S+MMM+CaPP }\end{array}$ & 1.6 & $4.102 \pm 0.044$ & $3.855 \pm 0.036$ & $4.960 \pm 0.046$ \\
\hline 67 & 13.92 & 816.7088 & $\mathrm{C}_{51} \mathrm{H}_{90} \mathrm{O}_{6}$ & $48: 4$ & LaPARA+MMARA & 1.5 & $0.046 \pm 0.001$ & $0.026 \pm 0.001$ & $0.024 \pm 0.001$ \\
\hline 68 & 13.93 & 842.7241 & $\mathrm{C}_{53} \mathrm{H}_{92} \mathrm{O}_{6}$ & $50: 5$ & $\mathrm{LaPDp}+\mathrm{MMDp}$ & 1.1 & $0.049 \pm 0.001$ & $0.020 \pm 0.001$ & $0.017 \pm 0.000$ \\
\hline 69 & 13.99 & 766.6937 & $\mathrm{C}_{47} \mathrm{H}_{88} \mathrm{O}_{6}$ & $44: 1$ & $\mathrm{CaPO}+\mathrm{LaMO}$ & 2.3 & $4.252 \pm 0.045$ & $4.272 \pm 0.030$ & $5.000 \pm 0.030$ \\
\hline 70 & 14.13 & 792.7099 & $\mathrm{C}_{49} \mathrm{H}_{90} \mathrm{O}_{6}$ & $46: 2$ & $\mathrm{CaOO}+\mathrm{MML}+\mathrm{LaPL}$ & 2.9 & $2.097 \pm 0.007$ & $2.383 \pm 0.094$ & $2.004 \pm 0.052$ \\
\hline 71 & 14.21 & 868.7394 & $\mathrm{C}_{55} \mathrm{H}_{94} \mathrm{O}_{6}$ & $52: 6$ & MOEPA+MLARA & 0.6 & $0.013 \pm 0.000$ & $0.006 \pm 0.000$ & $0.004 \pm 0.000$ \\
\hline 72 & 14.36 & 818.7248 & $\mathrm{C}_{51} \mathrm{H}_{92} \mathrm{O}_{6}$ & $48: 3$ & $\mathrm{LaOL}$ & 1.9 & $0.431 \pm 0.005$ & $0.289 \pm 0.011$ & $0.286 \pm 0.002$ \\
\hline 73 & 14.42 & 754.694 & $\mathrm{C}_{46} \mathrm{H}_{88} \mathrm{O}_{6}$ & $43: 0$ & $\begin{array}{l}\mathrm{CaPaS}+\mathrm{CaPMa}+\mathrm{UP} \\
\mathrm{P}+\mathrm{UMS}+\mathrm{UPaMa}+\mathrm{L} \\
\mathrm{aPaP}+\mathrm{LaTdS}+\mathrm{LaM} \\
\mathrm{Ma}+\mathrm{TdPaPa}+\mathrm{TdMP} \\
+\mathrm{TdTdMa}+\mathrm{MMPa}\end{array}$ & 2.8 & $0.607 \pm 0.007$ & $0.461 \pm 0.018$ & $0.561 \pm 0.008$ \\
\hline 74 & 14.49 & 894.7548 & $\mathrm{C}_{57} \mathrm{H}_{96} \mathrm{O}_{6}$ & $54: 7$ & PLEPA & 0.3 & $0.005 \pm 0.001$ & $0.002 \pm 0.001$ & $0.002 \pm 0.000$ \\
\hline 75 & 14.52 & 780.7099 & $\mathrm{C}_{48} \mathrm{H}_{90} \mathrm{O}_{6}$ & $45: 1$ & $\begin{array}{l}\mathrm{LaPaO}+\mathrm{MMMo}+\mathrm{U} \\
\mathrm{MaMo}+\mathrm{UPO}+\mathrm{CaMa} \\
\mathrm{O}\end{array}$ & 3.0 & $0.433 \pm 0.007$ & $0.379 \pm 0.015$ & $0.370 \pm 0.002$ \\
\hline 76 & 14.54 & 844.7408 & $\mathrm{C}_{53} \mathrm{H}_{94} \mathrm{O}_{6}$ & $50: 4$ & MOLn+MLL & 2.3 & $0.124 \pm 0.002$ & $0.062 \pm 0.003$ & $0.053 \pm 0.001$ \\
\hline 77 & 14.57 & 920.7707 & $\mathrm{C}_{59} \mathrm{H}_{98} \mathrm{O}_{6}$ & $56: 8$ & OLEPA & 0.6 & $0.004 \pm 0.000$ & $0.002 \pm 0.000$ & $0.001 \pm 0.000$ \\
\hline 78 & 14.61 & 868.7401 & $\mathrm{C}_{55} \mathrm{H}_{94} \mathrm{O}_{6}$ & $52: 6$ & MPDHA & 1.4 & $0.005 \pm 0.000$ & $0.001 \pm 0.000$ & $0.001 \pm 0.000$ \\
\hline 79 & 14.66 & 806.7237 & $\mathrm{C}_{50} \mathrm{H}_{92} \mathrm{O}_{6}$ & $47: 2$ & $\mathrm{LaMoO}+\mathrm{MPaL}$ & 0.6 & $0.118 \pm 0.003$ & $0.085 \pm 0.003$ & $0.080 \pm 0.002$ \\
\hline 80 & 14.89 & 832.7401 & $\mathrm{C}_{52} \mathrm{H}_{94} \mathrm{O}_{6}$ & $49: 3$ & $\begin{array}{l}\mathrm{PoPaL}+\mathrm{MoML}+\mathrm{Td} \\
\mathrm{OL}+\mathrm{MMaLn}+\mathrm{PaPL} \\
\mathrm{n}\end{array}$ & 1.5 & $0.043 \pm 0.001$ & $0.023 \pm 0.001$ & $0.019 \pm 0.002$ \\
\hline 81 & 14.95 & 768.7097 & $\mathrm{C}_{47} \mathrm{H}_{90} \mathrm{O}_{6}$ & $44: 0$ & $\begin{array}{l}\mathrm{CaPS}+\mathrm{LaMS}+\mathrm{LaPP} \\
+\mathrm{MPM}\end{array}$ & 2.7 & $3.602 \pm 0.043$ & $2.987 \pm 0.061$ & $3.694 \pm 0.021$ \\
\hline 82 & 14.99 & 844.7402 & $\mathrm{C}_{53} \mathrm{H}_{94} \mathrm{O}_{6}$ & $50: 4$ & MPARA & 1.6 & $0.078 \pm 0.002$ & $0.027 \pm 0.001$ & $0.025 \pm 0.001$ \\
\hline 83 & 15.02 & 870.7564 & $\mathrm{C}_{55} \mathrm{H}_{96} \mathrm{O}_{6}$ & $52: 5$ & MPDp+MOARA & 2.1 & $0.114 \pm 0.002$ & $0.034 \pm 0.001$ & $0.026 \pm 0.000$ \\
\hline 84 & 15.04 & 858.7547 & $\mathrm{C}_{54} \mathrm{H}_{96} \mathrm{O}_{6}$ & $51: 4$ & $\begin{array}{l}\mathrm{PMoLn}+\mathrm{PaOLn}+\mathrm{Pa} \\
\mathrm{LL}\end{array}$ & 0.2 & $0.018 \pm 0.001$ & $0.010 \pm 0.000$ & $0.008 \pm 0.000$ \\
\hline 85 & 15.07 & 794.7253 & $\mathrm{C}_{49} \mathrm{H}_{92} \mathrm{O}_{6}$ & $46: 1$ & $\begin{array}{l}\mathrm{LaSPo}+\mathrm{MPPo}+\mathrm{MM} \\
\mathrm{O}+\mathrm{LaPO}+\mathrm{CaSO}\end{array}$ & 2.6 & $4.010 \pm 0.017$ & $3.519 \pm 0.023$ & $3.875 \pm 0.041$ \\
\hline 86 & 15.10 & 896.7723 & $\mathrm{C}_{57} \mathrm{H}_{98} \mathrm{O}_{6}$ & $54: 6$ & MODp & 2.4 & $0.028 \pm 0.001$ & $0.007 \pm 0.001$ & $0.004 \pm 0.000$ \\
\hline 87 & 15.16 & 884.7702 & $\mathrm{C}_{56} \mathrm{H}_{98} \mathrm{O}_{6}$ & $53: 5$ & MoOLn+MoLLn & 0.1 & $0.003 \pm 0.000$ & $0.003 \pm 0.001$ & $0.001 \pm 0.001$ \\
\hline 88 & 15.22 & 820.7411 & $\mathrm{C}_{51} \mathrm{H}_{94} \mathrm{O}_{6}$ & $48: 2$ & $\begin{array}{l}\mathrm{MPoO}+\mathrm{PPoPo}+\mathrm{LaO} \\
\mathrm{O}+\mathrm{MPL}\end{array}$ & 2.7 & $2.458 \pm 0.016$ & $1.791 \pm 0.051$ & $1.567 \pm 0.020$ \\
\hline 89 & 15.25 & 780.7076 & $\mathrm{C}_{48} \mathrm{H}_{90} \mathrm{O}_{6}$ & $45: 1$ & LaPMo & 0.1 & $0.011 \pm 0.001$ & $0.006 \pm 0.000$ & $0.011 \pm 0.000$ \\
\hline 90 & 15.27 & 896.7702 & $\mathrm{C}_{57} \mathrm{H}_{98} \mathrm{O}_{6}$ & $54: 6$ & POEPA & 0.0 & $0.035 \pm 0.001$ & $0.016 \pm 0.002$ & $0.009 \pm 0.001$ \\
\hline 91 & 15.34 & 922.7871 & $\mathrm{C}_{59} \mathrm{H}_{100} \mathrm{O}_{6}$ & $56: 7$ & PLDp+OLARA & 1.3 & $0.010 \pm 0.003$ & $0.003 \pm 0.000$ & $0.002 \pm 0.000$ \\
\hline 92 & 15.37 & 846.7564 & $\mathrm{C}_{53} \mathrm{H}_{96} \mathrm{O}_{6}$ & $50: 3$ & $\mathrm{OML}+\mathrm{PoPL}$ & 2.2 & $0.751 \pm 0.021$ & $0.514 \pm 0.014$ & $0.416 \pm 0.003$ \\
\hline 93 & 15.38 & 948.8019 & $\mathrm{C}_{61} \mathrm{H}_{102} \mathrm{O}_{6}$ & $58: 8$ & OLDp & 0.4 & $0.003 \pm 0.000$ & $0.001 \pm 0.000$ & $0.001 \pm 0.000$ \\
\hline 94 & 15.48 & 782.725 & $\mathrm{C}_{48} \mathrm{H}_{92} \mathrm{O}_{6}$ & $45: 0$ & $\begin{array}{l}\mathrm{LaPMa}+\mathrm{LaPaS}+\mathrm{M} \\
\mathrm{MMa}+\mathrm{MPaP}+\mathrm{PaPaP} \\
\mathrm{a}\end{array}$ & 2.3 & $0.573 \pm 0.007$ & $0.311 \pm 0.017$ & $0.364 \pm 0.010$ \\
\hline 95 & 15.57 & 808.741 & $\mathrm{C}_{50} \mathrm{H}_{94} \mathrm{O}_{6}$ & $47: 1$ & $\mathrm{PaPaMo}+\mathrm{MPaO}$ & 2.6 & $0.580 \pm 0.010$ & $0.323 \pm 0.011$ & $0.309 \pm 0.004$ \\
\hline 96 & 15.58 & 872.7722 & $\mathrm{C}_{55} \mathrm{H}_{98} \mathrm{O}_{6}$ & $52: 4$ & POLn+PLL & 2.4 & $0.319 \pm 0.002$ & $0.196 \pm 0.007$ & $0.136 \pm 0.002$ \\
\hline
\end{tabular}




\begin{tabular}{|c|c|c|c|c|c|c|c|c|c|}
\hline 97 & 15.62 & 910.7867 & $\mathrm{C}_{58} \mathrm{H}_{100} \mathrm{O}_{6}$ & $55: 6$ & $\mathrm{PMoDp}+\mathrm{PaODp}$ & 0.9 & $0.006 \pm 0.000$ & $0.002 \pm 0.000$ & $0.001 \pm 0.000$ \\
\hline 98 & 15.66 & 898.7874 & $\mathrm{C}_{57} \mathrm{H}_{100} \mathrm{O}_{6}$ & $54: 5$ & OOLn+LLO & 1.8 & $0.123 \pm 0.002$ & $0.090 \pm 0.004$ & $0.053 \pm 0.001$ \\
\hline 99 & 15.68 & 834.7555 & $\mathrm{C}_{52} \mathrm{H}_{96} \mathrm{O}_{6}$ & $49: 2$ & $\begin{array}{l}\mathrm{PoPoMa}+\mathrm{PaMoMo}+ \\
\mathrm{PoPMo}+\mathrm{PoPaO}+\mathrm{M} \\
\mathrm{MaL}+\mathrm{PaPL}\end{array}$ & 1.2 & $0.248 \pm 0.001$ & $0.152 \pm 0.005$ & $0.116 \pm 0.001$ \\
\hline 100 & 15.76 & 922.7879 & $\mathrm{C}_{59} \mathrm{H}_{100} \mathrm{O}_{6}$ & $56: 7$ & PODHA & 2.3 & $0.013 \pm 0.001$ & $0.004 \pm 0.000$ & $0.002 \pm 0.000$ \\
\hline 101 & 15.88 & 860.7713 & $\mathrm{C}_{54} \mathrm{H}_{98} \mathrm{O}_{6}$ & $51: 3$ & $\begin{array}{l}\mathrm{PoMaL}+\mathrm{PMoL}+\mathrm{PaO} \\
\mathrm{L}+\mathrm{PMaLn}\end{array}$ & 1.3 & $0.103 \pm 0.000$ & $0.073 \pm 0.003$ & $0.055 \pm 0.001$ \\
\hline 102 & 15.98 & 886.7869 & $\mathrm{C}_{56} \mathrm{H}_{100} \mathrm{O}_{6}$ & $53: 4$ & $\mathrm{MaOLn}+\mathrm{MoOL}$ & 1.3 & $0.026 \pm 0.001$ & $0.025 \pm 0.002$ & $0.013 \pm 0.001$ \\
\hline 103 & 15.99 & 796.7409 & $\mathrm{C}_{49} \mathrm{H}_{94} \mathrm{O}_{6}$ & $46: 0$ & $\mathrm{LaPS}+\mathrm{MPP}+\mathrm{MMS}$ & 2.6 & $3.078 \pm 0.033$ & $1.988 \pm 0.066$ & $2.439 \pm 0.038$ \\
\hline 104 & 16.09 & 822.7564 & $\mathrm{C}_{51} \mathrm{H}_{96} \mathrm{O}_{6}$ & $48: 1$ & MPO & 2.3 & $1.976 \pm 0.318$ & $3.307 \pm 0.043$ & $3.745 \pm 0.023$ \\
\hline 105 & 16.11 & 898.7872 & $\mathrm{C}_{57} \mathrm{H}_{100} \mathrm{O}_{6}$ & $54: 5$ & POARA & 1.5 & $0.124 \pm 0.002$ & $0.050 \pm 0.001$ & $0.036 \pm 0.001$ \\
\hline 106 & 16.12 & 924.8043 & $\mathrm{C}_{59} \mathrm{H}_{102} \mathrm{O}_{6}$ & $56: 6$ & PODp & 3.1 & $0.138 \pm 0.002$ & $0.044 \pm 0.001$ & $0.026 \pm 0.000$ \\
\hline 107 & 16.21 & 848.7727 & $\mathrm{C}_{53} \mathrm{H}_{98} \mathrm{O}_{6}$ & $50: 2$ & $\mathrm{MOO}+\mathrm{PoPO}+\mathrm{PPL}$ & 3.0 & $3.287 \pm 0.051$ & $2.919 \pm 0.058$ & $2.356 \pm 0.033$ \\
\hline 108 & 16.22 & 950.8192 & $\mathrm{C}_{61} \mathrm{H}_{104} \mathrm{O}_{6}$ & $58: 7$ & OODp & 2.2 & $0.013 \pm 0.001$ & $0.006 \pm 0.000$ & $0.004 \pm 0.000$ \\
\hline 109 & 16.28 & 808.7386 & $\mathrm{C}_{50} \mathrm{H}_{94} \mathrm{O}_{6}$ & $47: 1$ & MPMo & -0.4 & $0.019 \pm 0.001$ & $0.007 \pm 0.001$ & $0.013 \pm 0.000$ \\
\hline 110 & 16.32 & 810.7558 & $\mathrm{C}_{50} \mathrm{H}_{96} \mathrm{O}_{6}$ & $47: 0$ & $\begin{array}{l}\mathrm{MPaS}+\mathrm{MPMa}+\mathrm{PaP} \\
\mathrm{P}\end{array}$ & 1.6 & $0.553 \pm 0.006$ & $0.274 \pm 0.011$ & $0.307 \pm 0.004$ \\
\hline 111 & 16.36 & 874.7886 & $\mathrm{C}_{55} \mathrm{H}_{100} \mathrm{O}_{6}$ & $52: 3$ & POL & 3.2 & $1.769 \pm 0.038$ & $1.457 \pm 0.048$ & $0.970 \pm 0.015$ \\
\hline 112 & 16.41 & 912.801 & $\mathrm{C}_{58} \mathrm{H}_{102} \mathrm{O}_{6}$ & $55: 5$ & PMaDp & -0.6 & $0.016 \pm 0.000$ & $0.006 \pm 0.000$ & $0.003 \pm 0.000$ \\
\hline 113 & 16.44 & 938.8182 & $\mathrm{C}_{60} \mathrm{H}_{104} \mathrm{O}_{6}$ & $57: 6$ & MaODp & 1.2 & $0.006 \pm 0.000$ & $0.001 \pm 0.000$ & $0.001 \pm 0.000$ \\
\hline 114 & 16.46 & 900.8034 & $\mathrm{C}_{57} \mathrm{H}_{102} \mathrm{O}_{6}$ & $54: 4$ & OOL & 2.1 & $0.545 \pm 0.003$ & $0.550 \pm 0.020$ & $0.298 \pm 0.002$ \\
\hline 115 & 16.57 & 836.7719 & $\mathrm{C}_{52} \mathrm{H}_{98} \mathrm{O}_{6}$ & $49: 1$ & $\begin{array}{l}\mathrm{MMaO}+\mathrm{PaPO}+\mathrm{PaM} \\
\text { aMo }\end{array}$ & 2.1 & $0.959 \pm 0.007$ & $0.532 \pm 0.022$ & $0.452 \pm 0.007$ \\
\hline 116 & 16.65 & 862.7876 & $\mathrm{C}_{54} \mathrm{H}_{100} \mathrm{O}_{6}$ & $51: 2$ & $\mathrm{PaOO}+\mathrm{PMoO}$ & 2.0 & $0.469 \pm 0.006$ & $0.390 \pm 0.017$ & $0.236 \pm 0.002$ \\
\hline 117 & 16.78 & 888.8026 & $\mathrm{C}_{56} \mathrm{H}_{102} \mathrm{O}_{6}$ & $53: 3$ & $\mathrm{MaOL}+\mathrm{MoOO}$ & 1.3 & $0.124 \pm 0.002$ & $0.134 \pm 0.007$ & $0.059 \pm 0.001$ \\
\hline 118 & 16.92 & 914.8184 & $\mathrm{C}_{58} \mathrm{H}_{104} \mathrm{O}_{6}$ & $55: 4$ & OLNe & 1.4 & $0.006 \pm 0.000$ & $0.005 \pm 0.000$ & $0.003 \pm 0.000$ \\
\hline 119 & 16.96 & 824.7729 & $\mathrm{C}_{51} \mathrm{H}_{98} \mathrm{O}_{6}$ & $48: 0$ & MPS & 3.3 & $2.360 \pm 0.040$ & $1.450 \pm 0.054$ & $1.711 \pm 0.010$ \\
\hline 120 & 16.97 & 926.8187 & $\mathrm{C}_{59} \mathrm{H}_{104} \mathrm{O}_{6}$ & $56: 5$ & PODt & 1.7 & $0.093 \pm 0.002$ & $0.032 \pm 0.001$ & $0.020 \pm 0.001$ \\
\hline 121 & 17.05 & 850.7881 & $\mathrm{C}_{53} \mathrm{H}_{100} \mathrm{O}_{6}$ & $50: 1$ & $\mathrm{MSO}+\mathrm{PPO}$ & 2.7 & $3.858 \pm 0.054$ & $3.252 \pm 0.075$ & $3.610 \pm 0.028$ \\
\hline 122 & 17.06 & 952.8349 & $\mathrm{C}_{61} \mathrm{H}_{106} \mathrm{O}_{6}$ & $58: 6$ & SODp & 2.2 & $0.012 \pm 0.000$ & $0.006 \pm 0.001$ & $0.004 \pm 0.000$ \\
\hline 123 & 17.14 & 876.8046 & $\mathrm{C}_{55} \mathrm{H}_{102} \mathrm{O}_{6}$ & $52: 2$ & $\mathrm{PSL}+\mathrm{POO}$ & 3.6 & $3.695 \pm 0.013$ & $3.646 \pm 0.082$ & $3.233 \pm 0.009$ \\
\hline 124 & 17.24 & 902.821 & $\mathrm{C}_{57} \mathrm{H}_{104} \mathrm{O}_{6}$ & $54: 3$ & OOO & 4.3 & $1.372 \pm 0.013$ & $1.852 \pm 0.079$ & $0.967 \pm 0.013$ \\
\hline 125 & 17.25 & 836.7717 & $\mathrm{C}_{52} \mathrm{H}_{98} \mathrm{O}_{6}$ & $49: 1$ & MSMo+PPMo & 1.8 & $0.016 \pm 0.000$ & $0.006 \pm 0.000$ & $0.012 \pm 0.000$ \\
\hline 126 & 17.26 & 940.8324 & $\mathrm{C}_{60} \mathrm{H}_{106} \mathrm{O}_{6}$ & $57: 5$ & MaSDp & -0.4 & $0.004 \pm 0.000$ & - & - \\
\hline 127 & 17.27 & 838.7886 & $\mathrm{C}_{52} \mathrm{H}_{100} \mathrm{O}_{6}$ & $49: 0$ & $\begin{array}{l}\mathrm{MMaS}+\mathrm{PaPS}+\mathrm{PaMa} \\
\mathrm{Ma}+\mathrm{PPMa}\end{array}$ & 3.3 & $0.390 \pm 0.005$ & $0.226 \pm 0.010$ & $0.220 \pm 0.003$ \\
\hline 128 & 17.42 & 864.8034 & $\mathrm{C}_{54} \mathrm{H}_{102} \mathrm{O}_{6}$ & $51: 1$ & $\mathrm{PMaO}$ & 2.2 & $0.690 \pm 0.007$ & $0.565 \pm 0.028$ & $0.360 \pm 0.004$ \\
\hline 129 & 17.51 & 928.8316 & $\mathrm{C}_{59} \mathrm{H}_{106} \mathrm{O}_{6}$ & $56: 4$ & SOEt+PODe & -1.3 & $0.027 \pm 0.000$ & $0.010 \pm 0.000$ & $0.006 \pm 0.000$ \\
\hline 130 & 17.52 & 890.818 & $\mathrm{C}_{56} \mathrm{H}_{104} \mathrm{O}_{6}$ & $53: 2$ & $\mathrm{MaOO}$ & 1.0 & $0.306 \pm 0.004$ & $0.334 \pm 0.016$ & $0.151 \pm 0.000$ \\
\hline 131 & 17.65 & 916.8336 & $\mathrm{C}_{58} \mathrm{H}_{106} \mathrm{O}_{6}$ & $55: 3$ & $\mathrm{OONe}$ & 0.9 & $0.019 \pm 0.000$ & $0.016 \pm 0.001$ & $0.008 \pm 0.001$ \\
\hline 132 & 17.86 & 852.8041 & $\mathrm{C}_{53} \mathrm{H}_{102} \mathrm{O}_{6}$ & $50: 0$ & PPS+SMS & 3.1 & $1.537 \pm 0.047$ & $1.199 \pm 0.048$ & $1.180 \pm 0.004$ \\
\hline
\end{tabular}




\begin{tabular}{|c|c|c|c|c|c|c|c|c|c|}
\hline 133 & 17.87 & 954.849 & $\mathrm{C}_{61} \mathrm{H}_{108} \mathrm{O}_{6}$ & $58: 5$ & SODt & 0.6 & $0.006 \pm 0.001$ & $0.003 \pm 0.000$ & $0.001 \pm 0.000$ \\
\hline 134 & 17.95 & 878.8203 & $\mathrm{C}_{55} \mathrm{H}_{104} \mathrm{O}_{6}$ & $52: 1$ & PSO & 3.6 & $2.418 \pm 0.023$ & $2.760 \pm 0.048$ & $2.304 \pm 0.010$ \\
\hline 135 & 18.03 & 904.8362 & $\mathrm{C}_{57} \mathrm{H}_{106} \mathrm{O}_{6}$ & $54: 2$ & $\mathrm{SOO}$ & 3.8 & $1.155 \pm 0.009$ & $1.731 \pm 0.058$ & $0.935 \pm 0.012$ \\
\hline 136 & 18.10 & 930.8488 & $\mathrm{C}_{59} \mathrm{H}_{108} \mathrm{O}_{6}$ & $56: 3$ & OOE & 0.5 & $0.025 \pm 0.000$ & $0.017 \pm 0.001$ & $0.011 \pm 0.000$ \\
\hline 137 & 18.14 & 864.8037 & $\mathrm{C}_{54} \mathrm{H}_{102} \mathrm{O}_{6}$ & $51: 1$ & $\mathrm{PMoS}$ & 2.6 & $0.007 \pm 0.000$ & $0.004 \pm 0.000$ & $0.005 \pm 0.000$ \\
\hline 138 & 18.24 & 890.8191 & $\mathrm{C}_{56} \mathrm{H}_{104} \mathrm{O}_{6}$ & $53: 2$ & $\mathrm{PONe}+\mathrm{MoSO}$ & 2.3 & $0.105 \pm 0.171$ & $0.004 \pm 0.000$ & $0.004 \pm 0.000$ \\
\hline 139 & 18.30 & 866.8195 & $\mathrm{C}_{54} \mathrm{H}_{104} \mathrm{O}_{6}$ & $51: 0$ & PMaS & 2.7 & $0.154 \pm 0.004$ & $0.130 \pm 0.005$ & $0.094 \pm 0.000$ \\
\hline 140 & 18.31 & 892.835 & $\mathrm{C}_{56} \mathrm{H}_{106} \mathrm{O}_{6}$ & $53: 1$ & $\mathrm{PONo}+\mathrm{MaSO}$ & 2.6 & $0.164 \pm 0.004$ & $0.187 \pm 0.006$ & $0.096 \pm 0.001$ \\
\hline 141 & 18.41 & 918.8501 & $\mathrm{C}_{58} \mathrm{H}_{108} \mathrm{O}_{6}$ & $55: 2$ & $\mathrm{SONe}+\mathrm{OONo}$ & 1.8 & $0.023 \pm 0.001$ & $0.020 \pm 0.001$ & $0.012 \pm 0.000$ \\
\hline 142 & 18.51 & 944.866 & $\mathrm{C}_{60} \mathrm{H}_{110} \mathrm{O}_{6}$ & $57: 3$ & $\mathrm{PLTe}+\mathrm{POTi}+\mathrm{PoOTe}$ & 2.0 & $0.004 \pm 0.000$ & $0.002 \pm 0.000$ & $0.002 \pm 0.000$ \\
\hline 143 & 18.72 & 880.8352 & $\mathrm{C}_{55} \mathrm{H}_{106} \mathrm{O}_{6}$ & $52: 0$ & PSS & 2.8 & $0.406 \pm 0.011$ & $0.563 \pm 0.032$ & $0.395 \pm 0.004$ \\
\hline 144 & 18.79 & 906.8504 & $\mathrm{C}_{57} \mathrm{H}_{108} \mathrm{O}_{6}$ & $54: 1$ & $\mathrm{OPA}+\mathrm{OSS}$ & 2.2 & $0.406 \pm 0.010$ & $0.639 \pm 0.031$ & $0.350 \pm 0.003$ \\
\hline 145 & 18.83 & 932.8656 & $\mathrm{C}_{59} \mathrm{H}_{110} \mathrm{O}_{6}$ & $56: 2$ & $\mathrm{PEE}+\mathrm{SOE}+\mathrm{PODo}$ & 1.6 & $0.032 \pm 0.000$ & $0.026 \pm 0.001$ & $0.018 \pm 0.000$ \\
\hline 146 & 18.91 & 958.881 & $\mathrm{C}_{61} \mathrm{H}_{112} \mathrm{O}_{6}$ & $58: 3$ & OLBe & 1.4 & $0.004 \pm 0.000$ & $0.002 \pm 0.000$ & $0.002 \pm 0.000$ \\
\hline 147 & 19.13 & 894.8497 & $\mathrm{C}_{56} \mathrm{H}_{108} \mathrm{O}_{6}$ & $53: 0$ & $\begin{array}{l}\mathrm{MPTa}+\mathrm{MSHe}+\mathrm{PaPa} \\
\mathrm{Ta}+\mathrm{PaMaHe}+\mathrm{PPHe} \\
+\mathrm{PSNo}+\mathrm{MaSS}+\mathrm{Ma} \\
\text { MaNo }\end{array}$ & 1.4 & $0.030 \pm 0.001$ & $0.029 \pm 0.001$ & $0.021 \pm 0.000$ \\
\hline 148 & 19.17 & 920.8654 & $\mathrm{C}_{58} \mathrm{H}_{110} \mathrm{O}_{6}$ & $55: 1$ & $\mathrm{POHe}+\mathrm{PPTe}$ & 1.4 & $0.023 \pm 0.000$ & $0.015 \pm 0.001$ & $0.013 \pm 0.000$ \\
\hline 149 & 19.19 & 946.8815 & $\mathrm{C}_{60} \mathrm{H}_{112} \mathrm{O}_{6}$ & $57: 2$ & POTe & 1.8 & $0.011 \pm 0.000$ & $0.007 \pm 0.000$ & $0.006 \pm 0.000$ \\
\hline 150 & 19.51 & 908.866 & $\mathrm{C}_{57} \mathrm{H}_{110} \mathrm{O}_{6}$ & $54: 0$ & PSA & 2.2 & $0.059 \pm 0.001$ & $0.065 \pm 0.004$ & $0.053 \pm 0.001$ \\
\hline 151 & 19.58 & 934.8809 & $\mathrm{C}_{59} \mathrm{H}_{112} \mathrm{O}_{6}$ & $56: 1$ & $\begin{array}{l}\mathrm{POBe}+\mathrm{PPLi}+\mathrm{SOA}+ \\
\mathrm{SSE}+\mathrm{PAE}+\mathrm{PPT}\end{array}$ & 1.2 & $0.031 \pm 0.001$ & $0.022 \pm 0.001$ & $0.018 \pm 0.000$ \\
\hline 152 & 19.62 & 960.8962 & $\mathrm{C}_{61} \mathrm{H}_{114} \mathrm{O}_{6}$ & $58: 2$ & POT & 0.9 & $0.008 \pm 0.000$ & $0.005 \pm 0.000$ & $0.004 \pm 0.000$ \\
\hline 153 & 19.69 & 986.9132 & $\mathrm{C}_{63} \mathrm{H}_{116} \mathrm{O}_{6}$ & $60: 3$ & OOT & 2.2 & $0.001 \pm 0.000$ & $0.001 \pm 0.000$ & $0.001 \pm 0.000$ \\
\hline 154 & 19.90 & 922.8796 & $\mathrm{C}_{58} \mathrm{H}_{112} \mathrm{O}_{6}$ & $55: 0$ & $\mathrm{MSTa}+\mathrm{PSHe}+\mathrm{PPTa}$ & -0.1 & $0.008 \pm 0.000$ & $0.005 \pm 0.000$ & $0.005 \pm 0.000$ \\
\hline 155 & 19.94 & 948.8968 & $\mathrm{C}_{60} \mathrm{H}_{114} \mathrm{O}_{6}$ & $57: 1$ & $\mathrm{POTa}+\mathrm{PSTe}+\mathrm{PPPb}$ & 1.5 & $0.009 \pm 0.000$ & $0.005 \pm 0.000$ & $0.005 \pm 0.000$ \\
\hline 156 & 19.97 & 974.9123 & $\mathrm{C}_{62} \mathrm{H}_{116} \mathrm{O}_{6}$ & $59: 2$ & POTe & 1.3 & $0.003 \pm 0.000$ & $0.002 \pm 0.000$ & $0.002 \pm 0.000$ \\
\hline 157 & 20.27 & 936.897 & $\mathrm{C}_{59} \mathrm{H}_{114} \mathrm{O}_{6}$ & $56: 0$ & $\begin{array}{l}\mathrm{MPHx}+\mathrm{MSLi}+\mathrm{PSBe} \\
+\mathrm{PPLi}\end{array}$ & 1.7 & $0.013 \pm 0.000$ & $0.008 \pm 0.000$ & $0.008 \pm 0.000$ \\
\hline 158 & 20.33 & 962.9129 & $\mathrm{C}_{61} \mathrm{H}_{116} \mathrm{O}_{6}$ & $58: 1$ & $\mathrm{POLi}+\mathrm{OSBe}$ & 1.9 & $0.009 \pm 0.000$ & $0.006 \pm 0.000$ & $0.006 \pm 0.000$ \\
\hline 159 & 20.39 & 988.9289 & $\mathrm{C}_{63} \mathrm{H}_{118} \mathrm{O}_{6}$ & $60: 2$ & $\begin{array}{l}\mathrm{POHc}+\mathrm{OLiO}+\mathrm{PLHx} \\
+\mathrm{SLiL}\end{array}$ & 2.3 & $0.003 \pm 0.000$ & $0.002 \pm 0.000$ & $0.002 \pm 0.000$ \\
\hline 160 & 20.60 & 950.9129 & $\mathrm{C}_{60} \mathrm{H}_{116} \mathrm{O}_{6}$ & $57: 0$ & $\mathrm{PSTa}+\mathrm{PPPc}$ & 2.0 & $0.004 \pm 0.000$ & $0.002 \pm 0.000$ & $0.002 \pm 0.000$ \\
\hline 161 & 20.66 & 976.9279 & $\mathrm{C}_{62} \mathrm{H}_{118} \mathrm{O}_{6}$ & $59: 1$ & $\mathrm{POPc}$ & 1.2 & $0.003 \pm 0.000$ & $0.002 \pm 0.000$ & $0.002 \pm 0.000$ \\
\hline 162 & 20.96 & 964.9273 & $\mathrm{C}_{61} \mathrm{H}_{118} \mathrm{O}_{6}$ & $58: 0$ & $\mathrm{PSLi}+\mathrm{PPHx}$ & 0.7 & $0.005 \pm 0.000$ & $0.003 \pm 0.000$ & $0.004 \pm 0.000$ \\
\hline 163 & 21.02 & 990.9449 & $\mathrm{C}_{63} \mathrm{H}_{120} \mathrm{O}_{6}$ & $60: 1$ & $\mathrm{POHx}+\mathrm{SLiO}$ & 2.6 & $0.004 \pm 0.000$ & $0.003 \pm 0.000$ & $0.003 \pm 0.000$ \\
\hline 164 & 21.09 & 1016.9604 & $\mathrm{C}_{65} \mathrm{H}_{122} \mathrm{O}_{6}$ & $62: 2$ & $\mathrm{HxOO}$ & 2.4 & $0.001 \pm 0.000$ & $0.001 \pm 0.000$ & $0.001 \pm 0.000$ \\
\hline 165 & 21.60 & 992.959 & $\mathrm{C}_{63} \mathrm{H}_{122} \mathrm{O}_{6}$ & $60: 0$ & PSHx+PPOc+SLiS & 1.1 & $0.001 \pm 0.000$ & $0.001 \pm 0.000$ & $0.001 \pm 0.000$ \\
\hline
\end{tabular}

${ }^{a}$ Values are presented as mass $\%$ in the form of means \pm standard deviation. ACN is acyl carbon number; DB is double bond. There was no distinction between the positions of $s n-1, s n-2$, and $s n-3$ in the identified triacylglycerols molecules.

${ }^{b}$ Values are experimental $\mathrm{m} / \mathrm{z}$ data; 
${ }^{c}$ Abbreviations are: Bu, butyric acid (C4:0); V, valeric acid (C5:0); Co, caproic acid (C6:0); Hp, heptanoic acid (C7:0); Cy, caprylic acid (C8:0); Pe, Pelargonic acid (C9:0); Ca, capric acid (C10:0); Dc, Decaenoic acid (10:1); U, Undecanoic acid (C11:0); La, lauric acid (12:0); Td, Tridecanoic acid (C13:0); M, myristic acid (C14:0); Pa, Pentadecanoic acid (C15:0); P, palmitic acid (C16:0); Po, Palmitoleic acid (C16:1); Ma, margaric acid (C17:0); Mo, margaroleic acid (C17:1); Hd, Heptadienoic acid (C17:2); S, stearic acid (C18:0); O, oleic acid (C18:1); L, linoleic acid (C18:2); Ln, linolenic acid (C18:3); No, Nonadecanoic acid (C19:0); Ne, Nonadecenoate acid (C19:1); A, eicosanoic acid (C20:0); E, eicosenoic acid (C20:1); Ed, eicosadienoic acid (C20:2); Et, Eicosatrienoic acid (C20:3); ARA, arachidonic acid (C20:4); EPA, eicosapentaenoic acid (C20:5); He, Heneicosanoic acid (C21:0); Be, behenic acid (C22:0); Do, Docosaenoic acid (C22:1); De, Docosaenotrienoic acid (C22:3); Dt, Docosatetraenoic acid (C22:4); Dp, docosapentaenoic acid (C22:5); DHA, docosahexaenoic acid (C22:6); Ta, tricosanoic acid (C23:0); Te, tricosenoic acid (C23:1); Ti, tricosadienoic acid (C23:2); Li, lignoceric acid (C24:0); T, Tetracarboxylic acid (C24:1); Pc, Pentacosanoic acid (C25:0); Pb, Pentacarbonic acid (C25:1); Hx, hexacosanoic acid (C26:0); Hc, Hexacosenoic acid (C26:1); Oc, Octacosanoic acid (C28:0). 
Table S2. Identified phospholipids (PLs) molecular species and their relative content (\% of total PLs) in goat colostrum, transitional and mature milk.

\begin{tabular}{|c|c|c|c|c|c|c|c|c|c|}
\hline $\mathrm{PLs}^{a}$ & Molecular species & $\begin{array}{l}\text { Retention } \\
\text { time }\end{array}$ & Formula & $\begin{array}{l}\mathrm{m} / \mathrm{z} \\
\text { observed }\end{array}$ & $\begin{array}{l}\text { Error } \\
(\mathrm{ppm})\end{array}$ & Ion $^{b}$ & Colostrum & Transitional & Mature \\
\hline \multirow[t]{8}{*}{ LPC } & $14: 0$ & 2.58 & $\mathrm{C}_{22} \mathrm{H}_{46} \mathrm{NO}_{7} \mathrm{P}$ & 512.2991 & -0.5 & {$[\mathrm{M}+\mathrm{FA}-\mathrm{H}]^{-}$} & $0.12 \pm 0.02$ & $0.08 \pm 0.04$ & $0.03 \pm 0.01$ \\
\hline & $16: 0$ & 3.54 & $\mathrm{C}_{24} \mathrm{H}_{50} \mathrm{NO}_{7} \mathrm{P}$ & 540.3304 & -0.5 & {$[\mathrm{M}+\mathrm{FA}-\mathrm{H}]^{-}$} & $0.70 \pm 0.05 \mathrm{ab}$ & $1.36 \pm 0.40 \mathrm{a}$ & $0.43 \pm 0.03 b$ \\
\hline & $16: 1$ & 2.81 & $\mathrm{C}_{24} \mathrm{H}_{48} \mathrm{NO}_{7} \mathrm{P}$ & 538.3142 & -1.5 & {$[\mathrm{M}+\mathrm{FA}-\mathrm{H}]^{-}$} & $0.03 \pm 0.01$ & $0.05 \pm 0.03$ & $0.02 \pm 0.01$ \\
\hline & $17: 0$ & 3.89 & $\mathrm{C}_{25} \mathrm{H}_{52} \mathrm{NO}_{7} \mathrm{P}$ & 554.3465 & 0.2 & {$[\mathrm{M}+\mathrm{FA}-\mathrm{H}]^{-}$} & $0.03 \pm 0.02 b$ & $0.11 \pm 0.03 \mathrm{a}$ & $0.03 \pm 0.02 b$ \\
\hline & $18: 0$ & 4.55 & $\mathrm{C}_{26} \mathrm{H}_{54} \mathrm{NO}_{7} \mathrm{P}$ & 568.3616 & -0.8 & {$[\mathrm{M}+\mathrm{FA}-\mathrm{H}]^{-}$} & $0.23 \pm 0.03 b$ & $0.42 \pm 0.08 \mathrm{a}$ & $0.12 \pm 0.02 b$ \\
\hline & $18: 1$ & 3.75 & $\mathrm{C}_{26} \mathrm{H}_{52} \mathrm{NO}_{7} \mathrm{P}$ & 566.3460 & -0.5 & {$[\mathrm{M}+\mathrm{FA}-\mathrm{H}]^{-}$} & $0.55 \pm 0.00$ & $1.53 \pm 0.61$ & $0.62 \pm 0.00$ \\
\hline & $18: 2$ & 2.26 & $\mathrm{C}_{26} \mathrm{H}_{50} \mathrm{NO}_{7} \mathrm{P}$ & 564.3307 & 0 & {$[\mathrm{M}+\mathrm{FA}-\mathrm{H}]^{-}$} & $0.20 \pm 0.04$ & $0.78 \pm 0.39$ & $0.31 \pm 0.02$ \\
\hline & $20: 4$ & 2.98 & $\mathrm{C}_{28} \mathrm{H}_{50} \mathrm{NO}_{7} \mathrm{P}$ & 588.3303 & -0.7 & {$[\mathrm{M}+\mathrm{FA}-\mathrm{H}]^{-}$} & $0.05 \pm 0.03$ & $0.08 \pm 0.04$ & $0.03 \pm 0.01$ \\
\hline \multirow[t]{33}{*}{$\mathrm{PC}$} & $10: 0-14: 0$ & 5.74 & $\mathrm{C}_{32} \mathrm{H}_{64} \mathrm{NO}_{8} \mathrm{P}$ & 666.4353 & 0.2 & {$[\mathrm{M}+\mathrm{FA}-\mathrm{H}]^{-}$} & $0.13 \pm 0.02 \mathrm{a}$ & $0.02 \pm 0.00 \mathrm{~b}$ & $0.09 \pm 0.02 \mathrm{ab}$ \\
\hline & $10: 0-16: 0 / 12: 0-14: 0$ & 6.41 & $\mathrm{C}_{34} \mathrm{H}_{68} \mathrm{NO}_{8} \mathrm{P}$ & 694.4661 & -0.6 & {$[\mathrm{M}+\mathrm{FA}-\mathrm{H}]^{-}$} & $0.27 \pm 0.00 \mathrm{a}$ & $0.07 \pm 0.01 \mathrm{c}$ & $0.17 \pm 0.02 b$ \\
\hline & $12: 0-16: 0 / 14: 0-14: 0$ & 7.16 & $\mathrm{C}_{36} \mathrm{H}_{72} \mathrm{NO}_{8} \mathrm{P}$ & 722.4973 & -0.7 & {$[\mathrm{M}+\mathrm{FA}-\mathrm{H}]^{-}$} & $0.51 \pm 0.12 \mathrm{a}$ & $0.18 \pm 0.06 \mathrm{~b}$ & $0.36 \pm 0.07 \mathrm{ab}$ \\
\hline & $10: 0-18: 1$ & 6.5 & $\mathrm{C}_{36} \mathrm{H}_{70} \mathrm{NO}_{8} \mathrm{P}$ & 720.4826 & 0.7 & {$[\mathrm{M}+\mathrm{FA}-\mathrm{H}]^{-}$} & $0.03 \pm 0.01$ & $0.02 \pm 0.00$ & $0.02 \pm 0.00$ \\
\hline & $14: 0-15: 0$ & 7.58 & $\mathrm{C}_{37} \mathrm{H}_{74} \mathrm{NO}_{8} \mathrm{P}$ & 736.5133 & -0.2 & {$[\mathrm{M}+\mathrm{FA}-\mathrm{H}]^{-}$} & $0.05 \pm 0.03$ & $0.02 \pm 0.01$ & $0.03 \pm 0.02$ \\
\hline & $14: 0-16: 0$ & 8.04 & $\mathrm{C}_{38} \mathrm{H}_{76} \mathrm{NO}_{8} \mathrm{P}$ & 750.5296 & 0.7 & {$[\mathrm{M}+\mathrm{FA}-\mathrm{H}]^{-}$} & $0.89 \pm 0.08 \mathrm{a}$ & $0.46 \pm 0.09 \mathrm{~b}$ & $0.73 \pm 0.03 \mathrm{a}$ \\
\hline & $15: 0-16: 0 / 14: 0-17: 0$ & 8.53 & $\mathrm{C}_{39} \mathrm{H}_{78} \mathrm{NO}_{8} \mathrm{P}$ & 764.5446 & -0.2 & {$[\mathrm{M}+\mathrm{FA}-\mathrm{H}]^{-}$} & $0.26 \pm 0.16$ & $0.21 \pm 0.19$ & $0.21 \pm 0.14$ \\
\hline & $16: 0-16: 0$ & 9.05 & $\mathrm{C}_{40} \mathrm{H}_{80} \mathrm{NO}_{8} \mathrm{P}$ & 778.5610 & 0.8 & {$[\mathrm{M}+\mathrm{FA}-\mathrm{H}]^{-}$} & $1.68 \pm 0.12$ & $1.61 \pm 0.26$ & $1.72 \pm 0.28$ \\
\hline & $14: 0-18: 1 / 16: 0-16: 1$ & 8.13 & $\mathrm{C}_{40} \mathrm{H}_{78} \mathrm{NO}_{8} \mathrm{P}$ & 776.5454 & 0.9 & {$[\mathrm{M}+\mathrm{FA}-\mathrm{H}]^{-}$} & $0.80 \pm 0.03 \mathrm{a}$ & $0.47 \pm 0.01 \mathrm{c}$ & $0.64 \pm 0.02 b$ \\
\hline & $14: 0-18: 2$ & 7.43 & $\mathrm{C}_{40} \mathrm{H}_{76} \mathrm{NO}_{8} \mathrm{P}$ & 774.5292 & 0.2 & {$[\mathrm{M}+\mathrm{FA}-\mathrm{H}]^{-}$} & $0.15 \pm 0.00$ & $0.08 \pm 0.02$ & $0.15 \pm 0.03$ \\
\hline & $16: 0-18: 0$ & 10.22 & $\mathrm{C}_{42} \mathrm{H}_{84} \mathrm{NO}_{8} \mathrm{P}$ & 806.5920 & 0.4 & {$[\mathrm{M}+\mathrm{FA}-\mathrm{H}]^{-}$} & $0.52 \pm 0.03$ & $0.66 \pm 0.08$ & $0.57 \pm 0.12$ \\
\hline & $16: 0-18: 1$ & 9.13 & $\mathrm{C}_{42} \mathrm{H}_{82} \mathrm{NO}_{8} \mathrm{P}$ & 804.5768 & 0.9 & {$[\mathrm{M}+\mathrm{FA}-\mathrm{H}]^{-}$} & $7.01 \pm 0.83$ & $7.05 \pm 0.13$ & $7.60 \pm 0.77$ \\
\hline & $16: 0-18: 2 / 18: 1-18: 1$ & 8.35 & $\mathrm{C}_{42} \mathrm{H}_{80} \mathrm{NO}_{8} \mathrm{P}$ & 802.5606 & 0.3 & {$[\mathrm{M}+\mathrm{FA}-\mathrm{H}]^{-}$} & $2.23 \pm 0.08 \mathrm{ab}$ & $1.90 \pm 0.13 b$ & $2.67 \pm 0.39 \mathrm{a}$ \\
\hline & $16: 0-18: 3$ & 7.7 & $\mathrm{C}_{42} \mathrm{H}_{78} \mathrm{NO}_{8} \mathrm{P}$ & 800.5452 & 0.7 & {$[\mathrm{M}+\mathrm{FA}-\mathrm{H}]^{-}$} & $0.17 \pm 0.05$ & $0.10 \pm 0.02$ & $0.14 \pm 0.01$ \\
\hline & $17: 0-18: 0 / 16: 0-19: 0$ & 10.66 & $\mathrm{C}_{43} \mathrm{H}_{86} \mathrm{NO}_{8} \mathrm{P}$ & 820.6083 & 1.3 & {$[\mathrm{M}+\mathrm{FA}-\mathrm{H}]^{-}$} & $0.08 \pm 0.04$ & $0.07 \pm 0.01$ & $0.07 \pm 0.02$ \\
\hline & $17: 0-18: 1 / 16: 0-19: 1$ & 9.53 & $\mathrm{C}_{43} \mathrm{H}_{84} \mathrm{NO}_{8} \mathrm{P}$ & 818.5921 & 0.5 & {$[\mathrm{M}+\mathrm{FA}-\mathrm{H}]^{-}$} & $0.32 \pm 0.00$ & $0.27 \pm 0.02$ & $0.31 \pm 0.08$ \\
\hline & $17: 1-18: 1 / 17: 0-18: 2$ & 8.71 & $\mathrm{C}_{43} \mathrm{H}_{82} \mathrm{NO}_{8} \mathrm{P}$ & 816.5765 & 0.6 & {$[\mathrm{M}+\mathrm{FA}-\mathrm{H}]^{-}$} & $0.19 \pm 0.07$ & $0.17 \pm 0.01$ & $0.17 \pm 0.00$ \\
\hline & 18:0-18:0/16:0-20:0 & 11.49 & $\mathrm{C}_{44} \mathrm{H}_{88} \mathrm{NO}_{8} \mathrm{P}$ & 834.6224 & -0.7 & {$[\mathrm{M}+\mathrm{FA}-\mathrm{H}]^{-}$} & $0.12 \pm 0.09$ & $0.16 \pm 0.14$ & $0.14 \pm 0.13$ \\
\hline & $18: 0-18: 1$ & 10.3 & $\mathrm{C}_{44} \mathrm{H}_{86} \mathrm{NO}_{8} \mathrm{P}$ & 832.6078 & 0.6 & {$[\mathrm{M}+\mathrm{FA}-\mathrm{H}]^{-}$} & $2.66 \pm 0.03$ & $2.58 \pm 0.24$ & $2.28 \pm 0.38$ \\
\hline & $18: 1-18: 1$ & 9.23 & $\mathrm{C}_{44} \mathrm{H}_{84} \mathrm{NO}_{8} \mathrm{P}$ & 830.5921 & 0.5 & {$[\mathrm{M}+\mathrm{FA}-\mathrm{H}]^{-}$} & $2.15 \pm 0.22$ & $2.22 \pm 0.08$ & $2.43 \pm 0.27$ \\
\hline & $18: 1-18: 2$ & 8.44 & $\mathrm{C}_{44} \mathrm{H}_{82} \mathrm{NO}_{8} \mathrm{P}$ & 828.5763 & 0.4 & {$[\mathrm{M}+\mathrm{FA}-\mathrm{H}]^{-}$} & $1.16 \pm 0.33$ & $1.15 \pm 0.04$ & $1.36 \pm 0.15$ \\
\hline & $16: 0-20: 4$ & 8.13 & $\mathrm{C}_{44} \mathrm{H}_{80} \mathrm{NO}_{8} \mathrm{P}$ & 826.5598 & -0.7 & {$[\mathrm{M}+\mathrm{FA}-\mathrm{H}]^{-}$} & $0.19 \pm 0.03 \mathrm{a}$ & $0.08 \pm 0.02 b$ & $0.08 \pm 0.02 b$ \\
\hline & $18: 2-18: 2$ & 7.72 & $\mathrm{C}_{44} \mathrm{H}_{80} \mathrm{NO}_{8} \mathrm{P}$ & 826.5605 & 0.1 & {$[\mathrm{M}+\mathrm{FA}-\mathrm{H}]^{-}$} & $0.16 \pm 0.08$ & $0.18 \pm 0.01$ & $0.23 \pm 0.01$ \\
\hline & $16: 0-20: 5$ & 7.53 & $\mathrm{C}_{44} \mathrm{H}_{78} \mathrm{NO}_{8} \mathrm{P}$ & 824.5450 & 0.4 & {$[\mathrm{M}+\mathrm{FA}-\mathrm{H}]^{-}$} & $0.05 \pm 0.00 \mathrm{a}$ & $0.02 \pm 0.00 \mathrm{~b}$ & $0.02 \pm 0.00 \mathrm{~b}$ \\
\hline & $18: 1-19: 1$ & 9.78 & $\mathrm{C}_{45} \mathrm{H}_{86} \mathrm{NO}_{8} \mathrm{P}$ & 844.6072 & -0.1 & {$[\mathrm{M}+\mathrm{FA}-\mathrm{H}]^{-}$} & $0.05 \pm 0.02$ & $0.03 \pm 0.01$ & $0.04 \pm 0.02$ \\
\hline & $20: 0-18: 1$ & 11.57 & $\mathrm{C}_{46} \mathrm{H}_{90} \mathrm{NO}_{8} \mathrm{P}$ & 860.6383 & -0.3 & {$[\mathrm{M}+\mathrm{FA}-\mathrm{H}]^{-}$} & $0.07 \pm 0.01$ & $0.06 \pm 0.00$ & $0.08 \pm 0.00$ \\
\hline & $18: 1-20: 1$ & 10.33 & $\mathrm{C}_{46} \mathrm{H}_{88} \mathrm{NO}_{8} \mathrm{P}$ & 858.6237 & 0.9 & {$[\mathrm{M}+\mathrm{FA}-\mathrm{H}]^{-}$} & $0.05 \pm 0.02$ & $0.03 \pm 0.01$ & $0.04 \pm 0.00$ \\
\hline & $18: 0-20: 3$ & 9.67 & $\mathrm{C}_{46} \mathrm{H}_{86} \mathrm{NO}_{8} \mathrm{P}$ & 856.6072 & -0.2 & {$[\mathrm{M}+\mathrm{FA}-\mathrm{H}]^{-}$} & $0.04 \pm 0.01 \mathrm{a}$ & $0.02 \pm 0.01 \mathrm{~b}$ & $0.02 \pm 0.00 \mathrm{~b}$ \\
\hline & $18: 0-20: 4$ & 9.15 & $\mathrm{C}_{46} \mathrm{H}_{84} \mathrm{NO}_{8} \mathrm{P}$ & 854.5921 & 0.6 & {$[\mathrm{M}+\mathrm{FA}-\mathrm{H}]^{-}$} & $0.08 \pm 0.01 \mathrm{a}$ & $0.06 \pm 0.01 \mathrm{ab}$ & $0.03 \pm 0.00 \mathrm{~b}$ \\
\hline & $18: 1-20: 4 / 22: 5-16: 0$ & 8.21 & $\mathrm{C}_{46} \mathrm{H}_{82} \mathrm{NO}_{8} \mathrm{P}$ & 852.5759 & -0.1 & {$[\mathrm{M}+\mathrm{FA}-\mathrm{H}]^{-}$} & $0.22 \pm 0.02$ & $0.15 \pm 0.02$ & $0.14 \pm 0.03$ \\
\hline & $18: 0-22: 5$ & 9.23 & $\mathrm{C}_{48} \mathrm{H}_{86} \mathrm{NO}_{8} \mathrm{P}$ & 880.6069 & -0.5 & {$[\mathrm{M}+\mathrm{FA}-\mathrm{H}]^{-}$} & $0.05 \pm 0.01 \mathrm{a}$ & $0.04 \pm 0.01 \mathrm{ab}$ & $0.02 \pm 0.00 \mathrm{~b}$ \\
\hline & $18: 1-22: 6$ & 7.92 & $\mathrm{C}_{48} \mathrm{H}_{82} \mathrm{NO}_{8} \mathrm{P}$ & 876.5759 & -0.1 & {$[\mathrm{M}+\mathrm{FA}-\mathrm{H}]^{-}$} & $0.02 \pm 0.01$ & $0.01 \pm 0.01$ & $0.02 \pm 0.00$ \\
\hline & $26: 0-18: 1$ & 15.14 & $\mathrm{C}_{52} \mathrm{H}_{102} \mathrm{NO}_{8} \mathrm{P}$ & 944.7329 & 0.4 & {$[\mathrm{M}+\mathrm{FA}-\mathrm{H}]^{-}$} & $0.01 \pm 0.01$ & - & $0.01 \pm 0.00$ \\
\hline
\end{tabular}




\begin{tabular}{|c|c|c|c|c|c|c|c|c|c|}
\hline \multirow[t]{3}{*}{$\mathrm{ePC}$} & $16: 0 e-16: 0$ & 9.98 & $\mathrm{C}_{40} \mathrm{H}_{82} \mathrm{NO}_{7} \mathrm{P}$ & 764.5805 & -0.7 & {$[\mathrm{M}+\mathrm{FA}-\mathrm{H}]^{-}$} & $0.05 \pm 0.01$ & $0.05 \pm 0.03$ & $0.03 \pm 0.02$ \\
\hline & $16: 0 \mathrm{e}-18: 1$ & 10.05 & $\mathrm{C}_{42} \mathrm{H}_{84} \mathrm{NO}_{7} \mathrm{P}$ & 790.5969 & 0.2 & {$[\mathrm{M}+\mathrm{FA}-\mathrm{H}]^{-}$} & $0.15 \pm 0.03$ & $0.22 \pm 0.02$ & $0.14 \pm 0.03$ \\
\hline & $18: 1 e-18: 1$ & 10.1 & $\mathrm{C}_{44} \mathrm{H}_{86} \mathrm{NO}_{7} \mathrm{P}$ & 816.6116 & -1 & {$[\mathrm{M}+\mathrm{FA}-\mathrm{H}]^{-}$} & $0.03 \pm 0.01 \mathrm{c}$ & $0.09 \pm 0.00 \mathrm{a}$ & $0.04 \pm 0.00 \mathrm{~b}$ \\
\hline \multirow[t]{32}{*}{ SM } & $\mathrm{d} 26: 1$ & 4.8 & $\mathrm{C}_{31} \mathrm{H}_{63} \mathrm{~N}_{2} \mathrm{O}_{6} \mathrm{P}$ & 635.4406 & 0.1 & {$[\mathrm{M}+\mathrm{FA}-\mathrm{H}]^{-}$} & $0.06 \pm 0.00 \mathrm{a}$ & $0.04 \pm 0.01 \mathrm{a}$ & $0.03 \pm 0.01 \mathrm{~b}$ \\
\hline & $\mathrm{d} 28: 1$ & 5.59 & $\mathrm{C}_{33} \mathrm{H}_{67} \mathrm{~N}_{2} \mathrm{O}_{6} \mathrm{P}$ & 663.4712 & -1 & {$[\mathrm{M}+\mathrm{FA}-\mathrm{H}]^{-}$} & $0.65 \pm 0.10 \mathrm{a}$ & $0.38 \pm 0.05 b$ & $0.37 \pm 0.09 \mathrm{~b}$ \\
\hline & $\mathrm{d} 28: 2$ & 5.13 & $\mathrm{C}_{33} \mathrm{H}_{65} \mathrm{~N}_{2} \mathrm{O}_{6} \mathrm{P}$ & 661.4568 & 0.9 & {$[\mathrm{M}+\mathrm{FA}-\mathrm{H}]^{-}$} & $0.02 \pm 0.00$ & $0.01 \pm 0.00$ & $0.01 \pm 0.00$ \\
\hline & $\mathrm{d} 29: 1$ & 5.83 & $\mathrm{C}_{34} \mathrm{H}_{69} \mathrm{~N}_{2} \mathrm{O}_{6} \mathrm{P}$ & 677.4871 & -0.6 & {$[\mathrm{M}+\mathrm{FA}-\mathrm{H}]^{-}$} & $0.08 \pm 0.02$ & $0.08 \pm 0.01$ & $0.05 \pm 0.01$ \\
\hline & $\mathrm{d} 30: 0$ & 6.59 & $\mathrm{C}_{35} \mathrm{H}_{73} \mathrm{~N}_{2} \mathrm{O}_{6} \mathrm{P}$ & 693.5186 & -0.4 & {$[\mathrm{M}+\mathrm{FA}-\mathrm{H}]^{-}$} & $0.06 \pm 0.01$ & $0.10 \pm 0.03$ & $0.05 \pm 0.00$ \\
\hline & $\mathrm{d} 30: 1$ & 6.24 & $\mathrm{C}_{35} \mathrm{H}_{71} \mathrm{~N}_{2} \mathrm{O}_{6} \mathrm{P}$ & 691.5031 & -0.1 & {$[\mathrm{M}+\mathrm{FA}-\mathrm{H}]^{-}$} & $0.37 \pm 0.01 \mathrm{a}$ & $0.24 \pm 0.08 \mathrm{ab}$ & $0.20 \pm 0.00 \mathrm{~b}$ \\
\hline & $\mathrm{d} 31: 1$ & 6.61 & $\mathrm{C}_{36} \mathrm{H}_{73} \mathrm{~N}_{2} \mathrm{O}_{6} \mathrm{P}$ & 705.5184 & -0.6 & {$[\mathrm{M}+\mathrm{FA}-\mathrm{H}]^{-}$} & $0.11 \pm 0.03$ & $0.04 \pm 0.03$ & $0.04 \pm 0.02$ \\
\hline & $\mathrm{d} 32: 0$ & 7.35 & $\mathrm{C}_{37} \mathrm{H}_{77} \mathrm{~N}_{2} \mathrm{O}_{6} \mathrm{P}$ & 721.5503 & 0.3 & {$[\mathrm{M}+\mathrm{FA}-\mathrm{H}]^{-}$} & $0.07 \pm 0.02$ & $0.09 \pm 0.05$ & $0.08 \pm 0.04$ \\
\hline & $\mathrm{d} 32: 1$ & 7.01 & $\mathrm{C}_{37} \mathrm{H}_{75} \mathrm{~N}_{2} \mathrm{O}_{6} \mathrm{P}$ & 719.5349 & 0.6 & {$[\mathrm{M}+\mathrm{FA}-\mathrm{H}]^{-}$} & $1.68 \pm 0.25$ & $1.10 \pm 0.20$ & $1.10 \pm 0.03$ \\
\hline & $\mathrm{d} 32: 2$ & 6.37 & $\mathrm{C}_{37} \mathrm{H}_{73} \mathrm{~N}_{2} \mathrm{O}_{6} \mathrm{P}$ & 717.5186 & -0.4 & {$[\mathrm{M}+\mathrm{FA}-\mathrm{H}]^{-}$} & $0.07 \pm 0.01 \mathrm{a}$ & $0.02 \pm 0.01 b$ & $0.02 \pm 0.00 \mathrm{~b}$ \\
\hline & d33:1 & 7.3 & $\mathrm{C}_{38} \mathrm{H}_{77} \mathrm{~N}_{2} \mathrm{O}_{6} \mathrm{P}$ & 733.5494 & -1 & {$[\mathrm{M}+\mathrm{FA}-\mathrm{H}]^{-}$} & $0.51 \pm 0.00$ & $0.37 \pm 0.10$ & $0.34 \pm 0.02$ \\
\hline & $\mathrm{d} 34: 0$ & 8.29 & $\mathrm{C}_{3}{ }_{9} \mathrm{H}_{81} \mathrm{~N}_{2} \mathrm{O}_{6} \mathrm{P}$ & 749.5815 & 0.1 & {$[\mathrm{M}+\mathrm{FA}-\mathrm{H}]^{-}$} & $0.09 \pm 0.00$ & $0.08 \pm 0.02$ & $0.08 \pm 0.01$ \\
\hline & $\mathrm{d} 34: 1$ & 7.89 & $\mathrm{C}_{39} \mathrm{H}_{79} \mathrm{~N}_{2} \mathrm{O}_{6} \mathrm{P}$ & 747.5662 & 0.6 & {$[\mathrm{M}+\mathrm{FA}-\mathrm{H}]^{-}$} & $4.40 \pm 0.15 \mathrm{a}$ & $2.86 \pm 0.85 \mathrm{ab}$ & $2.53 \pm 0.21 \mathrm{~b}$ \\
\hline & $\mathrm{d} 34: 2$ & 7.15 & $\mathrm{C}_{39} \mathrm{H}_{77} \mathrm{~N}_{2} \mathrm{O}_{6} \mathrm{P}$ & 745.5508 & 0.9 & {$[\mathrm{M}+\mathrm{FA}-\mathrm{H}]^{-}$} & $0.32 \pm 0.11$ & $0.19 \pm 0.08$ & $0.16 \pm 0.05$ \\
\hline & $\mathrm{d} 35: 0$ & 9.03 & $\mathrm{C}_{40} \mathrm{H}_{83} \mathrm{~N}_{2} \mathrm{O}_{6} \mathrm{P}$ & 763.5966 & -0.7 & {$[\mathrm{M}+\mathrm{FA}-\mathrm{H}]^{-}$} & $0.03 \pm 0.00$ & $0.04 \pm 0.00$ & $0.04 \pm 0.01$ \\
\hline & $\mathrm{d} 36: 0$ & 9.62 & $\mathrm{C}_{41} \mathrm{H}_{85} \mathrm{~N}_{2} \mathrm{O}_{6} \mathrm{P}$ & 777.6133 & 0.7 & {$[\mathrm{M}+\mathrm{FA}-\mathrm{H}]^{-}$} & $0.04 \pm 0.02$ & $0.04 \pm 0.01$ & $0.03 \pm 0.00$ \\
\hline & $\mathrm{d} 36: 1$ & 8.95 & $\mathrm{C}_{41} \mathrm{H}_{83} \mathrm{~N}_{2} \mathrm{O}_{6} \mathrm{P}$ & 775.5973 & 0.3 & {$[\mathrm{M}+\mathrm{FA}-\mathrm{H}]^{-}$} & $1.31 \pm 0.01 \mathrm{a}$ & $0.93 \pm 0.23 \mathrm{ab}$ & $0.55 \pm 0.17 b$ \\
\hline & $\mathrm{d} 36: 2$ & 8.12 & $\mathrm{C}_{41} \mathrm{H}_{81} \mathrm{~N}_{2} \mathrm{O}_{6} \mathrm{P}$ & 773.5808 & -0.8 & {$[\mathrm{M}+\mathrm{FA}-\mathrm{H}]^{-}$} & $0.27 \pm 0.05 \mathrm{a}$ & $0.18 \pm 0.00 \mathrm{ab}$ & $0.13 \pm 0.02 b$ \\
\hline & $\mathrm{d} 38: 1$ & 10.16 & $\mathrm{C}_{43} \mathrm{H}_{87} \mathrm{~N}_{2} \mathrm{O}_{6} \mathrm{P}$ & 803.6290 & 0.7 & {$[\mathrm{M}+\mathrm{FA}-\mathrm{H}]^{-}$} & $1.04 \pm 0.02 \mathrm{a}$ & $0.58 \pm 0.11 b$ & $0.43 \pm 0.04 \mathrm{~b}$ \\
\hline & d39:1 & 10.82 & $\mathrm{C}_{44} \mathrm{H}_{89} \mathrm{~N}_{2} \mathrm{O}_{6} \mathrm{P}$ & 817.6447 & 0.9 & {$[\mathrm{M}+\mathrm{FA}-\mathrm{H}]^{-}$} & $1.00 \pm 0.07 \mathrm{a}$ & $0.57 \pm 0.12 b$ & $0.48 \pm 0.03 b$ \\
\hline & $\mathrm{d} 39: 2$ & 9.54 & $\mathrm{C}_{44} \mathrm{H}_{87} \mathrm{~N}_{2} \mathrm{O}_{6} \mathrm{P}$ & 815.6290 & 0.8 & {$[\mathrm{M}+\mathrm{FA}-\mathrm{H}]^{-}$} & $0.04 \pm 0.01$ & $0.03 \pm 0.01$ & $0.03 \pm 0.00$ \\
\hline & $\mathrm{d} 40: 1$ & 11.41 & $\mathrm{C}_{45} \mathrm{H}_{91} \mathrm{~N}_{2} \mathrm{O}_{6} \mathrm{P}$ & 831.6600 & 0.4 & {$[\mathrm{M}+\mathrm{FA}-\mathrm{H}]^{-}$} & $2.31 \pm 0.06 \mathrm{a}$ & $1.37 \pm 0.21 b$ & $1.16 \pm 0.17 b$ \\
\hline & $\mathrm{d} 40: 2$ & 10.13 & $\mathrm{C}_{45} \mathrm{H}_{89} \mathrm{~N}_{2} \mathrm{O}_{6} \mathrm{P}$ & 829.6448 & 0.9 & {$[\mathrm{M}+\mathrm{FA}-\mathrm{H}]^{-}$} & $0.45 \pm 0.10 \mathrm{a}$ & $0.20 \pm 0.01 b$ & $0.17 \pm 0.04 b$ \\
\hline & $\mathrm{d} 41: 1$ & 11.84 & $\mathrm{C}_{46} \mathrm{H}_{93} \mathrm{~N}_{2} \mathrm{O}_{6} \mathrm{P}$ & 845.6756 & 0.3 & {$[\mathrm{M}+\mathrm{FA}-\mathrm{H}]^{-}$} & $2.31 \pm 0.19 \mathrm{a}$ & $1.38 \pm 0.15 b$ & $1.27 \pm 0.15 b$ \\
\hline & $\mathrm{d} 41: 2$ & 10.97 & $\mathrm{C}_{46} \mathrm{H}_{91} \mathrm{~N}_{2} \mathrm{O}_{6} \mathrm{P}$ & 843.6602 & 0.6 & {$[\mathrm{M}+\mathrm{FA}-\mathrm{H}]^{-}$} & $0.30 \pm 0.02$ & $0.19 \pm 0.15$ & $0.17 \pm 0.10$ \\
\hline & $\mathrm{d} 42: 1$ & 12.49 & $\mathrm{C}_{47} \mathrm{H}_{95} \mathrm{~N}_{2} \mathrm{O}_{6} \mathrm{P}$ & 859.6911 & 0.2 & {$[\mathrm{M}+\mathrm{FA}-\mathrm{H}]^{-}$} & $2.13 \pm 0.28 \mathrm{a}$ & $1.21 \pm 0.02 b$ & $1.14 \pm 0.06 \mathrm{~b}$ \\
\hline & $\mathrm{d} 42: 2$ & 11.33 & $\mathrm{C}_{47} \mathrm{H}_{93} \mathrm{~N}_{2} \mathrm{O}_{6} \mathrm{P}$ & 857.6762 & 1 & {$[\mathrm{M}+\mathrm{FA}-\mathrm{H}]^{-}$} & $1.04 \pm 0.02 \mathrm{a}$ & $0.51 \pm 0.10 \mathrm{~b}$ & $0.52 \pm 0.02 b$ \\
\hline & $\mathrm{d} 42: 3$ & 10.27 & $\mathrm{C}_{47} \mathrm{H}_{91} \mathrm{~N}_{2} \mathrm{O}_{6} \mathrm{P}$ & 855.6598 & 0.2 & {$[\mathrm{M}+\mathrm{FA}-\mathrm{H}]^{-}$} & $0.20 \pm 0.04 \mathrm{a}$ & $0.06 \pm 0.02 b$ & $0.05 \pm 0.01 \mathrm{~b}$ \\
\hline & $\mathrm{d} 43: 2$ & 11.76 & $\mathrm{C}_{48} \mathrm{H}_{95} \mathrm{~N}_{2} \mathrm{O}_{6} \mathrm{P}$ & 871.6918 & 1 & {$[\mathrm{M}+\mathrm{FA}-\mathrm{H}]^{-}$} & $0.15 \pm 0.01$ & $0.13 \pm 0.04$ & $0.10 \pm 0.01$ \\
\hline & $\mathrm{d} 43: 3$ & 10.9 & $\mathrm{C}_{48} \mathrm{H}_{93} \mathrm{~N}_{2} \mathrm{O}_{6} \mathrm{P}$ & 869.6754 & 0.1 & {$[\mathrm{M}+\mathrm{FA}-\mathrm{H}]^{-}$} & $0.02 \pm 0.01 \mathrm{a}$ & $0.01 \pm 0.00 \mathrm{~b}$ & $0.01 \pm 0.00 \mathrm{~b}$ \\
\hline & $\mathrm{d} 44: 1$ & 13.72 & $\mathrm{C}_{49} \mathrm{H}_{99} \mathrm{~N}_{2} \mathrm{O}_{6} \mathrm{P}$ & 887.7227 & 0.5 & {$[\mathrm{M}+\mathrm{FA}-\mathrm{H}]^{-}$} & $0.04 \pm 0.00$ & $0.03 \pm 0.00$ & $0.02 \pm 0.01$ \\
\hline & $\mathrm{d} 44: 2$ & 12.41 & $\mathrm{C}_{49} \mathrm{H}_{97} \mathrm{~N}_{2} \mathrm{O}_{6} \mathrm{P}$ & 885.7071 & 0.5 & {$[\mathrm{M}+\mathrm{FA}-\mathrm{H}]^{-}$} & $0.04 \pm 0.03$ & $0.02 \pm 0.00$ & $0.02 \pm 0.00$ \\
\hline \multirow[t]{11}{*}{ PE } & $12: 0-16: 0$ & 6.96 & $\mathrm{C}_{33} \mathrm{H}_{66} \mathrm{NO}_{8} \mathrm{P}$ & 634.4456 & 0.4 & {$[\mathrm{M}-\mathrm{H}]^{-}$} & $0.04 \pm 0.00 \mathrm{~b}$ & $0.03 \pm 0.00 \mathrm{~b}$ & $0.05 \pm 0.00 \mathrm{a}$ \\
\hline & $12: 0-18: 1 / 14: 0-16: 1$ & 7.06 & $\mathrm{C}_{35} \mathrm{H}_{68} \mathrm{NO}_{8} \mathrm{P}$ & 660.4604 & -0.8 & {$[\mathrm{M}-\mathrm{H}]^{-}$} & $0.04 \pm 0.00$ & $0.07 \pm 0.01$ & $0.07 \pm 0.00$ \\
\hline & $16: 0-16: 0$ & 8.68 & $\mathrm{C}_{37} \mathrm{H}_{74} \mathrm{NO}_{8} \mathrm{P}$ & 690.5073 & -0.9 & {$[\mathrm{M}-\mathrm{H}]^{-}$} & $0.10 \pm 0.01$ & $0.09 \pm 0.01$ & $0.10 \pm 0.03$ \\
\hline & $14: 0-18: 1$ & 7.88 & $\mathrm{C}_{37} \mathrm{H}_{72} \mathrm{NO}_{8} \mathrm{P}$ & 688.4928 & 0.8 & {$[\mathrm{M}-\mathrm{H}]^{-}$} & $0.54 \pm 0.06 \mathrm{ab}$ & $0.52 \pm 0.04 \mathrm{~b}$ & $0.66 \pm 0.04 \mathrm{a}$ \\
\hline & $14: 0-18: 2$ & 7.23 & $\mathrm{C}_{37} \mathrm{H}_{70} \mathrm{NO}_{8} \mathrm{P}$ & 686.4765 & -0.1 & {$[\mathrm{M}-\mathrm{H}]^{-}$} & $0.08 \pm 0.02 \mathrm{~b}$ & $0.11 \pm 0.00 \mathrm{ab}$ & $0.15 \pm 0.02 \mathrm{a}$ \\
\hline & $16: 0-17: 0$ & 8.98 & $\mathrm{C}_{38} \mathrm{H}_{76} \mathrm{NO}_{8} \mathrm{P}$ & 704.5242 & 0.9 & {$[\mathrm{M}-\mathrm{H}]^{-}$} & $0.03 \pm 0.01$ & $0.04 \pm 0.00$ & $0.03 \pm 0.00$ \\
\hline & $15: 0-18: 1 / 17: 1-16: 0$ & 8.32 & $\mathrm{C}_{38} \mathrm{H}_{74} \mathrm{NO}_{8} \mathrm{P}$ & 702.5085 & 0.9 & {$[\mathrm{M}-\mathrm{H}]^{-}$} & $0.13 \pm 0.00 \mathrm{~b}$ & $0.12 \pm 0.00 \mathrm{~b}$ & $0.16 \pm 0.01 \mathrm{a}$ \\
\hline & $15: 0-18: 2$ & 7.63 & $\mathrm{C}_{38} \mathrm{H}_{72} \mathrm{NO}_{8} \mathrm{P}$ & 700.4933 & 1.5 & {$[\mathrm{M}-\mathrm{H}]^{-}$} & $0.03 \pm 0.00 \mathrm{~b}$ & $0.03 \pm 0.01 \mathrm{~b}$ & $0.06 \pm 0.01 \mathrm{a}$ \\
\hline & $16: 0-18: 0$ & 9.7 & $\mathrm{C}_{39} \mathrm{H}_{78} \mathrm{NO}_{8} \mathrm{P}$ & 718.5399 & 0.9 & {$[\mathrm{M}-\mathrm{H}]^{-}$} & $0.05 \pm 0.00$ & $0.09 \pm 0.01$ & $0.06 \pm 0.02$ \\
\hline & $16: 0-18: 1$ & 8.79 & $\mathrm{C}_{39} \mathrm{H}_{76} \mathrm{NO}_{8} \mathrm{P}$ & 716.5242 & 0.9 & {$[\mathrm{M}-\mathrm{H}]^{-}$} & $5.25 \pm 0.24 b$ & $5.42 \pm 0.55 \mathrm{ab}$ & $6.46 \pm 0.10 \mathrm{a}$ \\
\hline & $16: 0-18: 2$ & 8.06 & $\mathrm{C}_{39} \mathrm{H}_{74} \mathrm{NO}_{8} \mathrm{P}$ & 714.5079 & 0 & {$[\mathrm{M}-\mathrm{H}]^{-}$} & $2.25 \pm 0.48 \mathrm{ab}$ & $1.92 \pm 0.04 \mathrm{~b}$ & $2.99 \pm 0.07 \mathrm{a}$ \\
\hline
\end{tabular}




\begin{tabular}{|c|c|c|c|c|c|c|c|c|c|}
\hline & $16: 0-18: 3$ & 7.47 & $\mathrm{C}_{39} \mathrm{H}_{72} \mathrm{NO}_{8} \mathrm{P}$ & 712.4918 & -0.7 & {$[\mathrm{M}-\mathrm{H}]^{-}$} & $0.16 \pm 0.03$ & $0.13 \pm 0.02$ & $0.18 \pm 0.02$ \\
\hline & $16: 1-18: 2$ & 7.34 & $\mathrm{C}_{39} \mathrm{H}_{72} \mathrm{NO}_{8} \mathrm{P}$ & 712.4928 & 0.8 & {$[\mathrm{M}-\mathrm{H}]^{-}$} & $0.02 \pm 0.02$ & $0.10 \pm 0.08$ & $0.14 \pm 0.09$ \\
\hline & $14: 0-20: 4$ & 7.09 & $\mathrm{C}_{39} \mathrm{H}_{70} \mathrm{NO}_{8} \mathrm{P}$ & 710.4766 & 0 & {$[\mathrm{M}-\mathrm{H}]^{-}$} & $0.02 \pm 0.00 \mathrm{a}$ & $0.01 \pm 0.00 \mathrm{~b}$ & $0.02 \pm 0.00 \mathrm{ab}$ \\
\hline & $17: 0-18: 0 / 16: 0-19: 0$ & 10.02 & $\mathrm{C}_{40} \mathrm{H}_{80} \mathrm{NO}_{8} \mathrm{P}$ & 732.5548 & -0.1 & {$[\mathrm{M}-\mathrm{H}]^{-}$} & $0.02 \pm 0.01$ & $0.03 \pm 0.01$ & $0.02 \pm 0.01$ \\
\hline & $17: 0-18: 1$ & 9.13 & $\mathrm{C}_{40} \mathrm{H}_{78} \mathrm{NO}_{8} \mathrm{P}$ & 730.5391 & -0.2 & {$[\mathrm{M}-\mathrm{H}]^{-}$} & $0.26 \pm 0.00$ & $0.25 \pm 0.01$ & $0.30 \pm 0.03$ \\
\hline & $17: 1-18: 1$ & 8.41 & $\mathrm{C}_{40} \mathrm{H}_{76} \mathrm{NO}_{8} \mathrm{P}$ & 728.5236 & 0 & {$[\mathrm{M}-\mathrm{H}]^{-}$} & $0.26 \pm 0.02 b$ & $0.33 \pm 0.00 \mathrm{a}$ & $0.37 \pm 0.03 \mathrm{a}$ \\
\hline & $17: 1-18: 2$ & 7.73 & $\mathrm{C}_{40} \mathrm{H}_{74} \mathrm{NO}_{8} \mathrm{P}$ & 726.5073 & -0.9 & {$[\mathrm{M}-\mathrm{H}]^{-}$} & $0.03 \pm 0.02$ & $0.04 \pm 0.00$ & $0.05 \pm 0.02$ \\
\hline & $18: 0-18: 0 / 20: 0-16: 0$ & 10.22 & $\mathrm{C}_{41} \mathrm{H}_{82} \mathrm{NO}_{8} \mathrm{P}$ & 746.5706 & 0.1 & {$[\mathrm{M}-\mathrm{H}]^{-}$} & $0.06 \pm 0.00$ & $0.08 \pm 0.00$ & $0.06 \pm 0.01$ \\
\hline & $18: 0-18: 1$ & 9.82 & $\mathrm{C}_{41} \mathrm{H}_{80} \mathrm{NO}_{8} \mathrm{P}$ & 744.5558 & 1.2 & {$[\mathrm{M}-\mathrm{H}]^{-}$} & $4.33 \pm 0.02 b$ & $5.76 \pm 0.36 \mathrm{a}$ & $4.97 \pm 0.17 \mathrm{a}$ \\
\hline & $18: 1-18: 1$ & 8.89 & $\mathrm{C}_{41} \mathrm{H}_{78} \mathrm{NO}_{8} \mathrm{P}$ & 742.5403 & 1.5 & {$[\mathrm{M}-\mathrm{H}]^{-}$} & $7.04 \pm 0.15 \mathrm{c}$ & $9.23 \pm 0.71 \mathrm{~b}$ & $11.26 \pm 0.34 \mathrm{a}$ \\
\hline & $18: 1-18: 2$ & 8.16 & $\mathrm{C}_{41} \mathrm{H}_{76} \mathrm{NO}_{8} \mathrm{P}$ & 740.5242 & 0.9 & {$[\mathrm{M}-\mathrm{H}]^{-}$} & $4.00 \pm 0.46 \mathrm{~b}$ & $5.18 \pm 0.89 \mathrm{ab}$ & $7.18 \pm 1.13 \mathrm{a}$ \\
\hline & $16: 0-20: 4$ & 7.9 & $\mathrm{C}_{41} \mathrm{H}_{74} \mathrm{NO}_{8} \mathrm{P}$ & 738.5083 & 0.5 & {$[\mathrm{M}-\mathrm{H}]^{-}$} & $0.59 \pm 0.08 \mathrm{a}$ & $0.23 \pm 0.01 \mathrm{~b}$ & $0.44 \pm 0.07 \mathrm{a}$ \\
\hline & $18: 1-18: 3 / 18: 2-18: 2$ & 7.57 & $\mathrm{C}_{41} \mathrm{H}_{74} \mathrm{NO}_{8} \mathrm{P}$ & 738.5083 & 0.5 & {$[\mathrm{M}-\mathrm{H}]^{-}$} & $0.43 \pm 0.04$ & $0.53 \pm 0.19$ & $0.76 \pm 0.22$ \\
\hline & $16: 0-20: 5$ & 7.35 & $\mathrm{C}_{41} \mathrm{H}_{72} \mathrm{NO}_{8} \mathrm{P}$ & 736.4928 & 0.8 & {$[\mathrm{M}-\mathrm{H}]^{-}$} & $0.09 \pm 0.00 \mathrm{a}$ & $0.04 \pm 0.00 \mathrm{~b}$ & $0.07 \pm 0.01 \mathrm{a}$ \\
\hline & $18: 1-19: 1$ & 9.38 & $\mathrm{C}_{42} \mathrm{H}_{80} \mathrm{NO}_{8} \mathrm{P}$ & 756.5552 & 0.5 & {$[\mathrm{M}-\mathrm{H}]^{-}$} & $0.10 \pm 0.00$ & $0.10 \pm 0.01$ & $0.10 \pm 0.00$ \\
\hline & $18: 1-20: 0$ & 10.88 & $\mathrm{C}_{43} \mathrm{H}_{84} \mathrm{NO}_{8} \mathrm{P}$ & 772.5867 & 0.6 & {$[\mathrm{M}-\mathrm{H}]^{-}$} & $0.14 \pm 0.00 \mathrm{~b}$ & $0.18 \pm 0.01 \mathrm{a}$ & $0.13 \pm 0.02 b$ \\
\hline & $18: 1-20: 1$ & 9.86 & $\mathrm{C}_{43} \mathrm{H}_{82} \mathrm{NO}_{8} \mathrm{P}$ & 770.5705 & 0 & {$[\mathrm{M}-\mathrm{H}]^{-}$} & $0.21 \pm 0.01$ & $0.22 \pm 0.00$ & $0.21 \pm 0.01$ \\
\hline & $18: 0-20: 3$ & 9.58 & $\mathrm{C}_{43} \mathrm{H}_{80} \mathrm{NO}_{8} \mathrm{P}$ & 768.5554 & 0.7 & {$[\mathrm{M}-\mathrm{H}]^{-}$} & $0.09 \pm 0.00$ & $0.08 \pm 0.04$ & $0.08 \pm 0.02$ \\
\hline & $18: 0-20: 4$ & 8.84 & $\mathrm{C}_{43} \mathrm{H}_{78} \mathrm{NO}_{8} \mathrm{P}$ & 766.5399 & 0.8 & {$[\mathrm{M}-\mathrm{H}]^{-}$} & $0.46 \pm 0.02 \mathrm{a}$ & $0.28 \pm 0.02 b$ & $0.28 \pm 0.01 b$ \\
\hline & $16: 0-22: 5 / 18: 1-20: 4$ & 7.97 & $\mathrm{C}_{43} \mathrm{H}_{76} \mathrm{NO}_{8} \mathrm{P}$ & 764.5240 & 0.6 & {$[\mathrm{M}-\mathrm{H}]^{-}$} & $1.11 \pm 0.37$ & $0.66 \pm 0.08$ & $0.71 \pm 0.02$ \\
\hline & $16: 0-22: 6$ & 7.67 & $\mathrm{C}_{43} \mathrm{H}_{74} \mathrm{NO}_{8} \mathrm{P}$ & 762.5101 & 2.8 & {$[\mathrm{M}-\mathrm{H}]^{-}$} & $0.06 \pm 0.00 \mathrm{a}$ & $0.04 \pm 0.00 \mathrm{~b}$ & $0.05 \pm 0.01 b$ \\
\hline & $18: 0-22: 5$ & 8.87 & $\mathrm{C}_{45} \mathrm{H}_{80} \mathrm{NO}_{8} \mathrm{P}$ & 792.5553 & 0.6 & {$[\mathrm{M}-\mathrm{H}]^{-}$} & $0.28 \pm 0.05$ & $0.22 \pm 0.00$ & $0.20 \pm 0.03$ \\
\hline & $18: 1-22: 4$ & 8.66 & $\mathrm{C}_{45} \mathrm{H}_{80} \mathrm{NO}_{8} \mathrm{P}$ & 792.5544 & -0.6 & {$[\mathrm{M}-\mathrm{H}]^{-}$} & $0.11 \pm 0.02$ & $0.08 \pm 0.01$ & $0.10 \pm 0.00$ \\
\hline & $18: 1-22: 5$ & 8.04 & $\mathrm{C}_{45} \mathrm{H}_{78} \mathrm{NO}_{8} \mathrm{P}$ & 790.5397 & 0.6 & {$[\mathrm{M}-\mathrm{H}]^{-}$} & $0.45 \pm 0.28$ & $0.38 \pm 0.12$ & $0.29 \pm 0.06$ \\
\hline ePE & $14: 0 \mathrm{e}-18: 1$ & 8.47 & $\mathrm{C}_{37} \mathrm{H}_{74} \mathrm{NO}_{7} \mathrm{P}$ & 674.5124 & -0.8 & {$[\mathrm{M}-\mathrm{H}]^{-}$} & $0.02 \pm 0.00$ & $0.02 \pm 0.01$ & $0.02 \pm 0.01$ \\
\hline & $16: 1 \mathrm{e}-17: 1$ & 8.85 & $\mathrm{C}_{38} \mathrm{H}_{74} \mathrm{NO}_{7} \mathrm{P}$ & 686.5125 & -0.8 & {$[\mathrm{M}-\mathrm{H}]^{-}$} & $0.08 \pm 0.01$ & $0.07 \pm 0.01$ & $0.05 \pm 0.01$ \\
\hline & $16: 0 e-18: 1$ & 9.53 & $\mathrm{C}_{39} \mathrm{H}_{78} \mathrm{NO}_{7} \mathrm{P}$ & 702.5444 & 0.2 & {$[\mathrm{M}-\mathrm{H}]^{-}$} & $0.13 \pm 0.02 b$ & $0.21 \pm 0.01 \mathrm{a}$ & $0.19 \pm 0.02 \mathrm{a}$ \\
\hline & $18: 2 \mathrm{e}-17: 1$ & 8.92 & $\mathrm{C}_{40} \mathrm{H}_{76} \mathrm{NO}_{7} \mathrm{P}$ & 712.5283 & -0.5 & {$[\mathrm{M}-\mathrm{H}]^{-}$} & $0.15 \pm 0.11$ & $0.13 \pm 0.08$ & $0.08 \pm 0.04$ \\
\hline & $18: 1 \mathrm{e}-18: 1$ & 10.45 & $\mathrm{C}_{41} \mathrm{H}_{80} \mathrm{NO}_{7} \mathrm{P}$ & 728.5605 & 0.8 & {$[\mathrm{M}-\mathrm{H}]^{-}$} & $2.31 \pm 0.29 \mathrm{ab}$ & $2.88 \pm 0.24 \mathrm{a}$ & $1.70 \pm 0.00 \mathrm{~b}$ \\
\hline & $18: 1 \mathrm{e}-18: 2$ & 9.59 & $\mathrm{C}_{41} \mathrm{H}_{78} \mathrm{NO}_{7} \mathrm{P}$ & 726.5448 & 0.7 & {$[\mathrm{M}-\mathrm{H}]^{-}$} & $3.74 \pm 0.18 \mathrm{a}$ & $3.64 \pm 0.21 \mathrm{a}$ & $2.36 \pm 0.02 b$ \\
\hline & $18: 2 \mathrm{e}-18: 2$ & 8.67 & $\mathrm{C}_{41} \mathrm{H}_{76} \mathrm{NO}_{7} \mathrm{P}$ & 724.5287 & 0.1 & {$[\mathrm{M}-\mathrm{H}]^{-}$} & $0.94 \pm 0.07 \mathrm{a}$ & $0.67 \pm 0.02 b$ & $0.51 \pm 0.06 \mathrm{~b}$ \\
\hline & $18: 2 \mathrm{e}-18: 3$ & 8.01 & $\mathrm{C}_{41} \mathrm{H}_{74} \mathrm{NO}_{7} \mathrm{P}$ & 722.5123 & -1 & {$[\mathrm{M}-\mathrm{H}]^{-}$} & $0.11 \pm 0.06$ & $0.04 \pm 0.01$ & $0.04 \pm 0.01$ \\
\hline & $18: 1 \mathrm{e}-22: 6$ & 9.08 & $\mathrm{C}_{45} \mathrm{H}_{78} \mathrm{NO}_{7} \mathrm{P}$ & 774.5450 & 0.8 & {$[\mathrm{M}-\mathrm{H}]^{-}$} & $0.09 \pm 0.02$ & $0.11 \pm 0.03$ & $0.04 \pm 0.01$ \\
\hline PG & $16: 0-18: 2$ & 6.79 & $\mathrm{C}_{40} \mathrm{H}_{75} \mathrm{O}_{10} \mathrm{P}$ & 745.5021 & -0.5 & {$[\mathrm{M}-\mathrm{H}]^{-}$} & $0.02 \pm 0.01$ & $0.02 \pm 0.00$ & $0.02 \pm 0.00$ \\
\hline & $18: 1-18: 2$ & 6.87 & $\mathrm{C}_{42} \mathrm{H}_{77} \mathrm{O}_{10} \mathrm{P}$ & 771.5177 & -0.6 & {$[\mathrm{M}-\mathrm{H}]^{-}$} & $0.01 \pm 0.01$ & $0.02 \pm 0.00$ & $0.01 \pm 0.00$ \\
\hline & $18: 2-18: 2$ & 6.42 & $\mathrm{C}_{42} \mathrm{H}_{75} \mathrm{O}_{10} \mathrm{P}$ & 769.5026 & 0.2 & {$[\mathrm{M}-\mathrm{H}]^{-}$} & $0.01 \pm 0.00$ & $0.02 \pm 0.00$ & $0.01 \pm 0.00$ \\
\hline PI & $14: 0-16: 0 / 15: 0-15: 0$ & 6.35 & $\mathrm{C}_{39} \mathrm{H}_{75} \mathrm{O}_{13} \mathrm{P}$ & 781.4872 & 0 & {$[\mathrm{M}-\mathrm{H}]^{-}$} & $0.04 \pm 0.01$ & $0.03 \pm 0.00$ & $0.04 \pm 0.01$ \\
\hline & $15: 0-16: 0$ & 6.53 & $\mathrm{C}_{40} \mathrm{H}_{77} \mathrm{O}_{13} \mathrm{P}$ & 795.5005 & -3 & {$[\mathrm{M}-\mathrm{H}]^{-}$} & $0.01 \pm 0.00$ & $0.01 \pm 0.00$ & $0.01 \pm 0.00$ \\
\hline & $16: 0-16: 0$ & 6.96 & $\mathrm{C}_{41} \mathrm{H}_{79} \mathrm{O}_{13} \mathrm{P}$ & 809.5182 & -0.4 & {$[\mathrm{M}-\mathrm{H}]^{-}$} & $0.12 \pm 0.01$ & $0.07 \pm 0.02$ & $0.07 \pm 0.03$ \\
\hline & $14: 0-18: 1$ & 6.42 & $\mathrm{C}_{41} \mathrm{H}_{77} \mathrm{O}_{13} \mathrm{P}$ & 807.5024 & -0.6 & {$[\mathrm{M}-\mathrm{H}]^{-}$} & $0.08 \pm 0.01$ & $0.08 \pm 0.00$ & $0.08 \pm 0.01$ \\
\hline & $14: 0-18: 2$ & 6.01 & $\mathrm{C}_{41} \mathrm{H}_{75} \mathrm{O}_{13} \mathrm{P}$ & 805.4859 & -1.7 & {$[\mathrm{M}-\mathrm{H}]^{-}$} & $0.01 \pm 0.01$ & $0.01 \pm 0.01$ & $0.01 \pm 0.01$ \\
\hline & $15: 0-18: 0 / 16: 0-17: 0$ & 7.18 & $\mathrm{C}_{42} \mathrm{H}_{81} \mathrm{O}_{13} \mathrm{P}$ & 823.5335 & -0.9 & {$[\mathrm{M}-\mathrm{H}]^{-}$} & $0.03 \pm 0.00$ & $0.03 \pm 0.01$ & $0.03 \pm 0.01$ \\
\hline & $15: 0-18: 1 / 16: 0-17: 1$ & 6.60 & $\mathrm{C}_{42} \mathrm{H}_{79} \mathrm{O}_{13} \mathrm{P}$ & 821.5181 & -0.6 & {$[\mathrm{M}-\mathrm{H}]^{-}$} & $0.03 \pm 0.03$ & $0.03 \pm 0.02$ & $0.03 \pm 0.03$ \\
\hline & $16: 0-18: 1$ & 7.04 & $\mathrm{C}_{43} \mathrm{H}_{81} \mathrm{O}_{13} \mathrm{P}$ & 835.5348 & 0.7 & {$[\mathrm{M}-\mathrm{H}]^{-}$} & $1.25 \pm 0.09 \mathrm{a}$ & $0.81 \pm 0.04 \mathrm{~b}$ & $0.93 \pm 0.01 b$ \\
\hline & $16: 0-18: 2$ & 6.56 & $\mathrm{C}_{43} \mathrm{H}_{79} \mathrm{O}_{13} \mathrm{P}$ & 833.5189 & 0.5 & {$[\mathrm{M}-\mathrm{H}]^{-}$} & $0.28 \pm 0.00 \mathrm{a}$ & $0.21 \pm 0.01 \mathrm{~b}$ & $0.27 \pm 0.01 \mathrm{a}$ \\
\hline & $16: 0-18: 3$ & 6.17 & $\mathrm{C}_{43} \mathrm{H}_{77} \mathrm{O}_{13} \mathrm{P}$ & 831.5025 & -0.5 & {$[\mathrm{M}-\mathrm{H}]^{-}$} & $0.02 \pm 0.02$ & $0.02 \pm 0.01$ & $0.01 \pm 0.01$ \\
\hline
\end{tabular}




\begin{tabular}{|c|c|c|c|c|c|c|c|c|c|}
\hline & $17: 0-18: 0$ & 7.90 & $\mathrm{C}_{44} \mathrm{H}_{85} \mathrm{O}_{13} \mathrm{P}$ & 851.5647 & -0.9 & {$[\mathrm{M}-\mathrm{H}]^{-}$} & $0.03 \pm 0.01$ & $0.04 \pm 0.01$ & $0.03 \pm 0.00$ \\
\hline & $17: 0-18: 1$ & 7.40 & $\mathrm{C}_{44} \mathrm{H}_{83} \mathrm{O}_{13} \mathrm{P}$ & 849.5497 & -0.1 & {$[\mathrm{M}-\mathrm{H}]^{-}$} & $0.11 \pm 0.02$ & $0.11 \pm 0.04$ & $0.08 \pm 0.03$ \\
\hline & $18: 0-18: 1$ & 7.78 & $\mathrm{C}_{45} \mathrm{H}_{85} \mathrm{O}_{13} \mathrm{P}$ & 863.5667 & 1.4 & {$[\mathrm{M}-\mathrm{H}]^{-}$} & $3.54 \pm 0.21 \mathrm{~b}$ & $4.81 \pm 0.23 \mathrm{a}$ & $4.54 \pm 0.12 \mathrm{a}$ \\
\hline & $18: 0-18: 2$ & 7.21 & $\mathrm{C}_{45} \mathrm{H}_{83} \mathrm{O}_{13} \mathrm{P}$ & 861.5502 & 0.3 & {$[\mathrm{M}-\mathrm{H}]^{-}$} & $2.27 \pm 0.65$ & $3.02 \pm 0.85$ & $4.24 \pm 0.27$ \\
\hline & $18: 1-18: 2$ & 6.64 & $\mathrm{C}_{45} \mathrm{H}_{81} \mathrm{O}_{13} \mathrm{P}$ & 859.5346 & 0.4 & {$[\mathrm{M}-\mathrm{H}]^{-}$} & $0.38 \pm 0.03 \mathrm{~b}$ & $0.46 \pm 0.03 b$ & $0.61 \pm 0.02 \mathrm{a}$ \\
\hline & $16: 0-20: 4$ & 6.47 & $\mathrm{C}_{45} \mathrm{H}_{79} \mathrm{O}_{13} \mathrm{P}$ & 857.5182 & -0.4 & {$[\mathrm{M}-\mathrm{H}]^{-}$} & $0.21 \pm 0.08$ & $0.10 \pm 0.03$ & $0.08 \pm 0.02$ \\
\hline & $18: 2-18: 2 / 18: 1-18: 3$ & 6.24 & $\mathrm{C}_{45} \mathrm{H}_{79} \mathrm{O}_{13} \mathrm{P}$ & 857.5180 & -0.6 & {$[\mathrm{M}-\mathrm{H}]^{-}$} & $0.03 \pm 0.03$ & $0.04 \pm 0.03$ & $0.04 \pm 0.02$ \\
\hline & $16: 0-20: 5$ & 6.12 & $\mathrm{C}_{45} \mathrm{H}_{77} \mathrm{O}_{13} \mathrm{P}$ & 855.5026 & -0.3 & {$[\mathrm{M}-\mathrm{H}]^{-}$} & $0.03 \pm 0.02$ & $0.01 \pm 0.01$ & $0.01 \pm 0.01$ \\
\hline & $18: 0-20: 3$ & 7.63 & $\mathrm{C}_{47} \mathrm{H}_{85} \mathrm{O}_{13} \mathrm{P}$ & 887.5656 & 0.1 & {$[\mathrm{M}-\mathrm{H}]^{-}$} & $0.08 \pm 0.01$ & $0.07 \pm 0.01$ & $0.06 \pm 0.02$ \\
\hline & $18: 0-20: 4$ & 7.11 & $\mathrm{C}_{47} \mathrm{H}_{83} \mathrm{O}_{13} \mathrm{P}$ & 885.5503 & 0.5 & {$[\mathrm{M}-\mathrm{H}]^{-}$} & $0.55 \pm 0.06 \mathrm{ab}$ & $0.65 \pm 0.11 \mathrm{a}$ & $0.39 \pm 0.06 b$ \\
\hline & $18: 1-20: 4$ & 6.55 & $\mathrm{C}_{47} \mathrm{H}_{81} \mathrm{O}_{13} \mathrm{P}$ & 883.5335 & -0.8 & {$[\mathrm{M}-\mathrm{H}]^{-}$} & $0.21 \pm 0.00 \mathrm{a}$ & $0.22 \pm 0.01 \mathrm{a}$ & $0.13 \pm 0.00 \mathrm{~b}$ \\
\hline & $18: 1-20: 5$ & 6.18 & $\mathrm{C}_{47} \mathrm{H}_{79} \mathrm{O}_{13} \mathrm{P}$ & 881.5186 & 0.1 & {$[\mathrm{M}-\mathrm{H}]^{-}$} & $0.04 \pm 0.00$ & $0.03 \pm 0.01$ & $0.03 \pm 0.00$ \\
\hline & $18: 0-22: 5$ & 7.13 & $\mathrm{C}_{49} \mathrm{H}_{85} \mathrm{O}_{13} \mathrm{P}$ & 911.5649 & -0.7 & {$[\mathrm{M}-\mathrm{H}]^{-}$} & $0.06 \pm 0.02$ & $0.03 \pm 0.01$ & $0.02 \pm 0.00$ \\
\hline PS & $16: 0-18: 1$ & 7.55 & $\mathrm{C}_{40} \mathrm{H}_{76} \mathrm{NO}_{10} \mathrm{P}$ & 760.5130 & -0.6 & {$[\mathrm{M}-\mathrm{H}]^{-}$} & $0.21 \pm 0.02 \mathrm{a}$ & $0.13 \pm 0.01 \mathrm{~b}$ & $0.15 \pm 0.02 \mathrm{ab}$ \\
\hline & $16: 0-18: 2$ & 7.10 & $\mathrm{C}_{40} \mathrm{H}_{74} \mathrm{NO}_{10} \mathrm{P}$ & 758.4983 & 0.7 & {$[\mathrm{M}-\mathrm{H}]^{-}$} & $0.06 \pm 0.02$ & $0.03 \pm 0.01$ & $0.05 \pm 0.02$ \\
\hline & $18: 0-18: 1$ & 8.33 & $\mathrm{C}_{42} \mathrm{H}_{80} \mathrm{NO}_{10} \mathrm{P}$ & 788.5454 & 0.9 & {$[\mathrm{M}-\mathrm{H}]^{-}$} & $3.26 \pm 0.14$ & $3.41 \pm 0.25$ & $3.13 \pm 0.60$ \\
\hline & $18: 1-18: 1 / 18: 0-18: 2$ & 7.65 & $\mathrm{C}_{42} \mathrm{H}_{78} \mathrm{NO}_{10} \mathrm{P}$ & 786.5297 & 0.8 & {$[\mathrm{M}-\mathrm{H}]^{-}$} & $2.27 \pm 0.03$ & $2.34 \pm 0.24$ & $2.70 \pm 0.15$ \\
\hline & $18: 1-18: 2$ & 7.26 & $\mathrm{C}_{42} \mathrm{H}_{76} \mathrm{NO}_{10} \mathrm{P}$ & 784.5129 & -0.7 & {$[\mathrm{M}-\mathrm{H}]^{-}$} & $0.53 \pm 0.18$ & $0.57 \pm 0.17$ & $0.69 \pm 0.13$ \\
\hline & $18: 0-22: 4$ & 8.24 & $\mathrm{C}_{46} \mathrm{H}_{82} \mathrm{NO}_{10} \mathrm{P}$ & 838.5598 & -0.6 & {$[\mathrm{M}-\mathrm{H}]^{-}$} & $0.17 \pm 0.00 \mathrm{a}$ & $0.06 \pm 0.01 \mathrm{~b}$ & $0.07 \pm 0.02 \mathrm{~b}$ \\
\hline & $18: 0-22: 5$ & 7.58 & $\mathrm{C}_{46} \mathrm{H}_{80} \mathrm{NO}_{10} \mathrm{P}$ & 836.5442 & -0.6 & {$[\mathrm{M}-\mathrm{H}]^{-}$} & $0.61 \pm 0.03 \mathrm{a}$ & $0.36 \pm 0.00 \mathrm{~b}$ & $0.33 \pm 0.05 b$ \\
\hline LPE & $16: 0$ & 3.47 & $\mathrm{C}_{21} \mathrm{H}_{44} \mathrm{NO}_{7} \mathrm{P}$ & 452.2786 & 0.8 & {$[\mathrm{M}-\mathrm{H}]^{-}$} & $0.20 \pm 0.08$ & $0.38 \pm 0.22$ & $0.10 \pm 0.04$ \\
\hline & $17: 1$ & 3.19 & $\mathrm{C}_{22} \mathrm{H}_{44} \mathrm{NO}_{7} \mathrm{P}$ & 464.2783 & 0.1 & {$[\mathrm{M}-\mathrm{H}]^{-}$} & $0.01 \pm 0.00$ & $0.05 \pm 0.02$ & $0.01 \pm 0.00$ \\
\hline & $18: 1$ & 3.69 & $\mathrm{C}_{23} \mathrm{H}_{46} \mathrm{NO}_{7} \mathrm{P}$ & 478.2936 & -0.6 & {$[\mathrm{M}-\mathrm{H}]^{-}$} & $0.64 \pm 0.07$ & $2.21 \pm 1.10$ & $0.96 \pm 0.03$ \\
\hline & $18: 2$ & 2.85 & $\mathrm{C}_{23} \mathrm{H}_{44} \mathrm{NO}_{7} \mathrm{P}$ & 476.2780 & -0.5 & {$[\mathrm{M}-\mathrm{H}]^{-}$} & $0.03 \pm 0.03$ & $0.06 \pm 0.08$ & $0.04 \pm 0.06$ \\
\hline & $20: 3$ & 3.57 & $\mathrm{C}_{25} \mathrm{H}_{46} \mathrm{NO}_{7} \mathrm{P}$ & 502.2938 & -0.2 & {$[\mathrm{M}-\mathrm{H}]^{-}$} & $0.01 \pm 0.00$ & $0.05 \pm 0.02$ & $0.01 \pm 0.00$ \\
\hline & $20: 4$ & 2.93 & $\mathrm{C}_{25} \mathrm{H}_{44} \mathrm{NO}_{7} \mathrm{P}$ & 500.2778 & -1 & {$[\mathrm{M}-\mathrm{H}]^{-}$} & $0.06 \pm 0.00$ & $0.10 \pm 0.03$ & $0.04 \pm 0.01$ \\
\hline & $22: 4$ & 3.68 & $\mathrm{C}_{27} \mathrm{H}_{48} \mathrm{NO}_{7} \mathrm{P}$ & 528.3100 & 0.8 & {$[\mathrm{M}-\mathrm{H}]^{-}$} & $0.02 \pm 0.00 \mathrm{~b}$ & $0.06 \pm 0.01 \mathrm{a}$ & $0.02 \pm 0.01 b$ \\
\hline & $22: 5$ & 3.10 & $\mathrm{C}_{27} \mathrm{H}_{46} \mathrm{NO}_{7} \mathrm{P}$ & 526.2943 & 0.7 & {$[\mathrm{M}-\mathrm{H}]^{-}$} & $0.05 \pm 0.02$ & $0.20 \pm 0.09$ & $0.06 \pm 0.00$ \\
\hline LPI & $18: 0$ & 3.35 & $\mathrm{C}_{27} \mathrm{H}_{53} \mathrm{O}_{12} \mathrm{P}$ & 599.3203 & 0.2 & {$[\mathrm{M}-\mathrm{H}]^{-}$} & $0.01 \pm 0.00$ & $0.04 \pm 0.00$ & $0.01 \pm 0.00$ \\
\hline & $18: 1$ & 2.63 & $\mathrm{C}_{27} \mathrm{H}_{51} \mathrm{O}_{12} \mathrm{P}$ & 597.3043 & -0.5 & {$[\mathrm{M}-\mathrm{H}]^{-}$} & $0.01 \pm 0.00 \mathrm{~b}$ & $0.07 \pm 0.01 \mathrm{a}$ & $0.02 \pm 0.00 \mathrm{~b}$ \\
\hline & $18: 2$ & 1.60 & $\mathrm{C}_{27} \mathrm{H}_{49} \mathrm{O}_{12} \mathrm{P}$ & 595.2889 & 0.1 & {$[\mathrm{M}-\mathrm{H}]^{-}$} & $0.02 \pm 0.00 \mathrm{c}$ & $0.19 \pm 0.00 \mathrm{a}$ & $0.07 \pm 0.00 \mathrm{~b}$ \\
\hline & $20: 4$ & 1.59 & $\mathrm{C}_{29} \mathrm{H}_{49} \mathrm{O}_{12} \mathrm{P}$ & 619.2890 & 0.2 & {$[\mathrm{M}-\mathrm{H}]^{-}$} & $0.02 \pm 0.00 \mathrm{~b}$ & $0.14 \pm 0.00 \mathrm{a}$ & $0.04 \pm 0.00 \mathrm{~b}$ \\
\hline PA & $18: 0-18: 1$ & 8.85 & $\mathrm{C}_{39} \mathrm{H}_{75} \mathrm{O}_{8} \mathrm{P}$ & 701.5130 & 0.4 & {$[\mathrm{M}-\mathrm{H}]^{-}$} & $0.10 \pm 0.03$ & $0.10 \pm 0.03$ & $0.09 \pm 0.03$ \\
\hline & $18: 1-18: 1$ & 8.13 & $\mathrm{C}_{39} \mathrm{H}_{73} \mathrm{O}_{8} \mathrm{P}$ & 699.4971 & 0.1 & {$[\mathrm{M}-\mathrm{H}]^{-}$} & $0.08 \pm 0.00$ & $0.09 \pm 0.01$ & $0.09 \pm 0.01$ \\
\hline & $18: 1-18: 2$ & 7.41 & $\mathrm{C}_{39} \mathrm{H}_{71} \mathrm{O}_{8} \mathrm{P}$ & 697.4812 & -0.3 & {$[\mathrm{M}-\mathrm{H}]^{-}$} & $0.02 \pm 0.02$ & $0.04 \pm 0.04$ & $0.03 \pm 0.02$ \\
\hline
\end{tabular}

${ }^{a}$ Abbreviations are: LPC, lyso-phosphatidylcholine; PC, phosphatidylcholine; SM, sphingomyelin; ePC, ether phosphatidylcholine; LPE, lyso-phosphatidylethanolamine; PE, phosphatidylethanolamine; ePE, ether phosphatidylethanolamine; PI, phosphatidylinositol; PS, phosphatidylserine; PG, phosphatidylglycerol; PA, phosphatidic acid; LPI, lyso-phosphatidylinositol.

${ }^{b}$ Abbreviation is: FA, formic acid. 